\title{
Microwave-Assisted Synthesis under Solvent-Free Conditions of (E)-2-(Benzo[d]thiazol-2-yl)-3-arylacrylonitriles
}

\author{
Jorge E. Trilleras, ${ }^{*}, a$ Kelly J. Velasquez, ${ }^{a}$ Dency J. Pacheco, ${ }^{a}$ Jairo Quiroga ${ }^{b}$ and \\ Alejandro Ortiz ${ }^{b}$
}

\author{
${ }^{a}$ Grupo de Investigación en Compuestos Heterocíclicos, Programa de Química, \\ Facultad de Ciencias Básicas, Universidad del Atlántico, Km 7 Antigua vía Puerto Colombia, \\ Barranquilla-Atlántico, Colombia
}

${ }^{b}$ Grupo de Investigación de Compuestos Heterocíclicos, Departamento de Química, Universidad del Valle, A. A 25360 Cali, Colombia

\begin{abstract}
Uma série de (E)-2-(benzo[d]tiazol-2-il)-3-arilacrilonitrilas foi sintetizada pela condensação de Knoevenagel assistida por microondas, na ausência de solvente, partindo do correspondente 2-(benzo $[d]$ tiazo-2-il)acetonitrila e aldeídos aromáticos, contendo tanto grupos doadores de elétrons, como retiradores. Os tempos de reação foram consideravelmente curtos e os produtos, obtidos em rendimentos moderados (50-75\%) e boa pureza. A configuração da dupla ligação da acrilonitrila não pôde ser estabelecida por métodos comuns de RMN. No entanto, estudos teóricos sugerem que nesses compostos o isômero $E$ é mais estável do que o $Z$, o que está de acordo com algumas evidências experimentais.
\end{abstract}

A series of (E)-2-(benzo[d]thiazol-2-yl)-3-arylacrylonitriles was synthesized by microwave assisted Knoevenagel condensation under solvent-free conditions from the corresponding 2-(benzo[ $[d]$ thiazol-2-yl)acetonitrile and aromatic aldehydes with electrondonating/electronwithdrawing groups. The reaction times were considerably short and the products obtained in moderate yields (50 to $75 \%$ ) and good purity. The configuration of the acrylonitrile double bond could not be established by regular NMR methods. However, theoretical studies suggest that the $E$ isomer is more stable than $Z$, which is in good agreement with some experimental evidences.

Keywords: acrylonitriles, 2-(benzo[ $d]$ thiazol-2-yl)acetonitrile, solvent-free reaction, irradiation microwave, Knoevenagel condensation, benzaldehydes

\section{Introduction}

Acetonitrile derivatives are convenient precursors which have been extensively utilized in heterocyclic synthesis. Several reactions were developed in the last decades for which the reactivity of hetarylacetonitriles towards diverse reagents was exploited for the synthesis of nitrogen bridged heterocycles. ${ }^{1-3}$ From the point of view for biological activities, acetonitrile derivatives are useful intermediates and subunits for the development of molecules having pharmaceutical or biological interests. ${ }^{4-6}$ Nitrogen containing heteroaromatic compounds have received considerable attention in the literature over the years. From the synthetic point of the view, it is known

*e-mail: jorgetrilleras@mail.uniatlantico.edu.co that heteroaromatic acetonitrile derivatives undergo Knoevenagel condensation reactions with arylaldehydes to yield 3-aryl-2hetarylacrylonitriles. ${ }^{1-3,5-9}$ Substituted acrylonitriles have been found to possess interesting biological properties, among them, antifungal and antitumor activities. ${ }^{7,10,11}$ Although acrylonitrile derivatives are an-easy-to synthesize template, a great diversity of the methods is available in literature that describe their synthesis under different conditions making use of conventional thermal energy and microwave irradiation conditions..$^{1-3,12-17}$ Typically, Knoevenagel adducts have been obtained under conventional heating conditions for extended periods of reaction in the presence of catalytic amounts of base, obtaining generally from good to very low yields. ${ }^{18-23}$ Condensation reactions leading to heterocyclic systems have been performed with great success under 
microwave irradiation in "dry media" reducing substantially the reaction times and increasing product yields. ${ }^{24-26}$ Because of the advantages that microwave irradiation has over conventional reflux methods, we present, herein, the Knoevenagel synthesis of 3-arylacrylonitriles by the reaction of 2-(benzo[d]thiazol-2-yl)acetonitrile with different substituted arylaldehydes. The synthesis has been assisted by microwave irradiation and under solventfree conditions. When compared with other methods, this one has better yields, milder reaction conditions and easy work-up, in addition to inexpensive reagents, and an environmentally friendly procedure.

\section{Results and Discussion}

Microwave enhanced synthesis of $(E)$-2-(benzo $[d]$ thiazol-2-yl)-3-arylacrylonitriles $\mathbf{3 a - j}$ was carried out under solvent-free conditions using different substituted arylaldehydes $\mathbf{2}$ as shown in Scheme 1 and Table 1 .

The reactions proceeded quite well with different substituted aldehydes with electrondonating/electronwithdrawing groups giving moderate yields (50 to 75\%).

The condensation between 2-(benzo[ $d]$ thiazol-2-yl) acetonitrile $\mathbf{1}$ and different substituted arylaldehydes 2 yields (E)-2-(benzo[ $[$ ] thiazol-2-yl)-3-arylacrylonitriles $\mathbf{3}$ in "dry media" conditions in excellent purities and moderate yields. Reaction times were reduced to minutes rather than hours when compared with conventional thermal synthesis. ${ }^{18-23}$ This type of condensation reaction represents a great potential for the synthesis of heterocyclic compounds with possible biological activity. The fast reaction times, combined with the easy purification and improved yields make this method highly suitable. The synthesis of products consists in irradiating the heterogeneous mixture of 2-(benzo[ $[d]$ thiazol-2-yl)acetonitrile 1 with different substituted arylaldehydes $\mathbf{2}$ through reactions that are conducted with neat solid reagents without catalysts; elevation of the temperature had some positive effects and improved the yield of the target product. Due to the dipolar polarization phenomenon, the greater the polarity of a molecule the more pronounced the microwave effect when the rise in temperature is considered. In terms of reactivity, the specific effect has therefore to be considered according to the reaction mechanism and particularly with regard to how the polarity of the system is altered during the progress of the reaction. ${ }^{12}$

In initial attempts, we carried out the reaction under solvent-free conditions during 15-20 min of microwave irradiation in a commercial domestic oven, and then the reactions conditions were optimized in a focused microwaves reactor (CEM Discover TM) twice. In order to improve our initial attempts, different reaction times, power and temperatures were studied for the preparation of $(E)$-2(benzo[ $d]$ thiazol-2-yl)-3-arylacrylonitriles 3 . The reaction
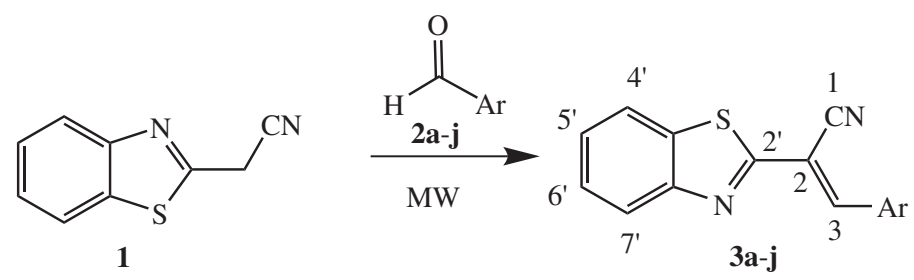

Scheme 1. Microwave irradiation synthesis under solvent-free conditions of (E)-2-(benzo[d]thiazol-2-yl)-3-arylacrylonitriles 3a-j.

Table 1. Knoevenagel reaction between 2-(benzo $[d]$ thiazol-2-yl)acetonitrile $(\mathbf{1})$ and aromatic aldehydes (2a-j)

\begin{tabular}{|c|c|c|c|c|}
\hline & \multirow{2}{*}{$\mathrm{Ar}$} & \multicolumn{3}{|c|}{ Data reported in literature for compounds $\mathbf{3} \mathbf{a}-\mathbf{j}^{18-23}$} \\
\hline & & time reaction / min & yield / (\%) & $\mathrm{mp} /\left({ }^{\circ} \mathrm{C}\right)$ \\
\hline $\mathbf{a}$ & $\mathrm{C}_{6} \mathrm{H}_{5}$ & $15 / 180$ & $70 / 46$ & $116-118 / 121-123$ \\
\hline $\mathbf{b}$ & $4-\mathrm{H}_{3} \mathrm{C}-\mathrm{C}_{6} \mathrm{H}_{4}$ & $15 / 180$ & $70 / 54$ & $140-142 / 147-148$ \\
\hline c & $4-\mathrm{O}_{2} \mathrm{~N}-\mathrm{C}_{6} \mathrm{H}_{4}$ & $20 / 240$ & $50 / 50$ & $170-172 / 177-180$ \\
\hline d & $4-\mathrm{Cl}-\mathrm{C}_{6} \mathrm{H}_{4}$ & $20 / 240$ & $75 / 51$ & $144-148 / 148-150$ \\
\hline e & $4-\mathrm{Br}-\mathrm{C}_{6} \mathrm{H}_{4}$ & $20 / 180$ & $50 / 54$ & $138-140 / 143-145$ \\
\hline $\mathbf{f}$ & $4-\mathrm{F}-\mathrm{C}_{6} \mathrm{H}_{4}$ & 10 & 50 & $150-152$ \\
\hline $\mathbf{g}$ & $4-\mathrm{F}_{3} \mathrm{C}-\mathrm{C}_{6} \mathrm{H}_{4}$ & $10 / 180$ & $75 / 56$ & $122-124 / 128-133$ \\
\hline $\mathbf{h}$ & $4-\mathrm{H}_{3} \mathrm{COC}_{6} \mathrm{H}_{4}$ & $15 / 180$ & $50 / 62$ & 130-dec./143-144 \\
\hline i & $3,4,5-$ tri- $\mathrm{H}_{3} \mathrm{CO}-\mathrm{C}_{6} \mathrm{H}_{2}$ & 15 & 55 & $140-142$ \\
\hline $\mathbf{j}$ & $3,4-\mathrm{OCH}_{2} \mathrm{O}-\mathrm{C}_{6} \mathrm{H}_{3}$ & $15 / 180$ & $50 / 40$ & $192-194 / 200-205$ \\
\hline
\end{tabular}


carried under the maximum power $150 \mathrm{~W}$ during 10-20 min at a controlled temperature of $473 \mathrm{~K}$ and 250 psi proved to be the most satisfactory. An increase in the irradiation time did not affect the yields.

All compounds were characterized on the basis of their elemental analyses, mass and NMR spectral data. The whole carbon skeleton was assigned using ${ }^{13} \mathrm{C}$ NMR spectra, (including distortionless enhancement by polarization transfer (DEPT)) and two dimensional HSQC (heteronuclear single quantum correlation), and HMBC (heteronuclear multiple bond correlation) experiments. The (E)-2-(benzo[ $d]$ thiazol-2-yl)-3-arylacrylonitriles $\mathbf{3 a - j}$ were were characterized by a very polarized carbon-carbon double bond (C2-C3), which leads to chemical shifts from 102.2 to $109.5 \mathrm{ppm}$ for $\mathrm{C} 2$ and 143.2 to $146.9 \mathrm{ppm}$ for $\mathrm{C} 3$; the signal of $\mathrm{CN}$ at $\delta$ values from 115.6 to $117.3 \mathrm{ppm}$; from ${ }^{1} \mathrm{H} \mathrm{NMR}$, a singlet between 8.12 and 8.30 ppm for $\mathrm{H} 3$ (Figure 1).

The ${ }^{13} \mathrm{C}$ NMR spectra of all other compounds of the series retain similar signals, differing only in those corresponding to the aryl ring. Regarding to mass spectra, all products $\mathbf{3}$ exhibit a similar pattern of fragmentation, showing the molecular peak along with a typical loss of the substituents of each aryl group.

The configuration of the acrylonitrile double bond could not be established by NMR methods. However, studies have shown that for these compounds the $E$ isomer is more stable than the $Z$. This result can be explained by the steric and electronic repulsions between the aromatic and heteroaromatic groups. ${ }^{27}$ In previous research on these
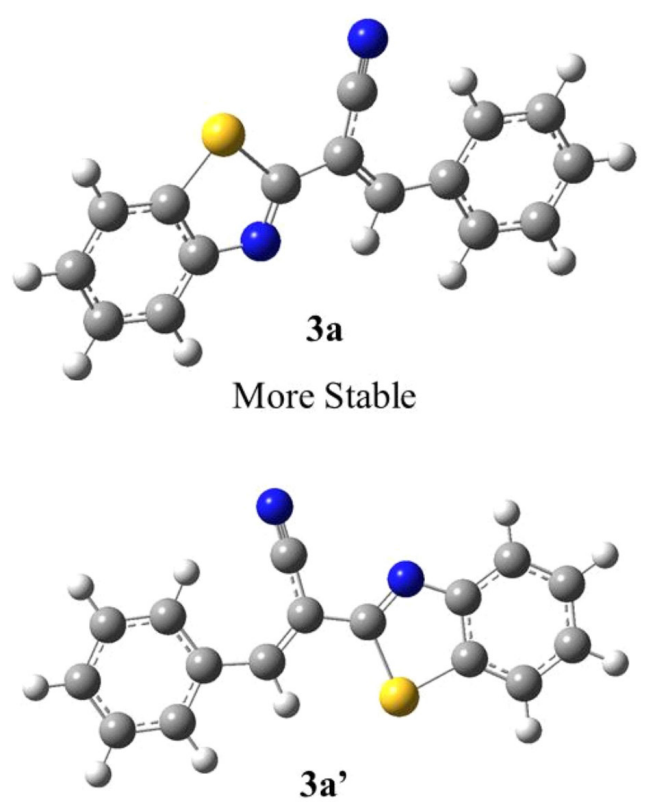

$+0.07206 \mathrm{eV}$

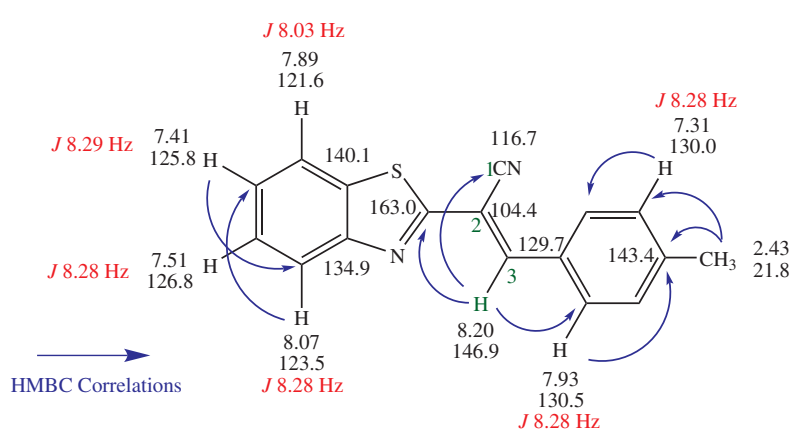

Figure 1. Structural formula of $(E)-2-($ benzo $[d]$ thiazol-2-yl)-3-ptolylacrylonitrile $\mathbf{3 b}$.

3-arylacrylonitrile derivatives it has also been observed preference for the $E$ isomer. ${ }^{7,13-15}$ Compound (3a) and $(E)$ 3-phenyl-2-(thiophen-2-yl)acrylonitrile (4) previously reported by us, ${ }^{7}$ might have a number of conformations due to free rotation around the double bond; the most stable conformations are shown in Figure 2 and apparently the lowest energy structure is favored by planarity of the molecule around the double bond mentioned; this fact was calculated by means theoretical methods like DFT using a B3LYP functional and 3-21G as basis set.

When performing an analysis of net atomic charges of the most stable conformers, it was found that for conformer 3a, the presence of a hydrogen bond between the $\mathrm{H} 3$ and $\mathrm{N}$, is the driving force for greater stability and planarity; these two atoms are at a distance of $2.399 \AA$, the $\mathrm{H} 3$ also presents a net charge of $0.26556 \mathrm{e}$ and a valence of 0.73151
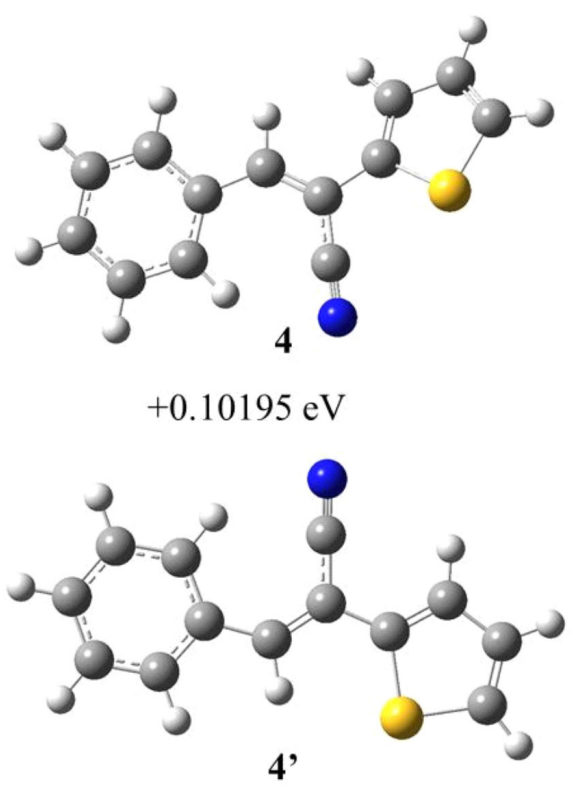

More Stable

Figure 2. Lowest energy structures calculated for $\mathbf{3 a}$ and $\mathbf{4}$. 


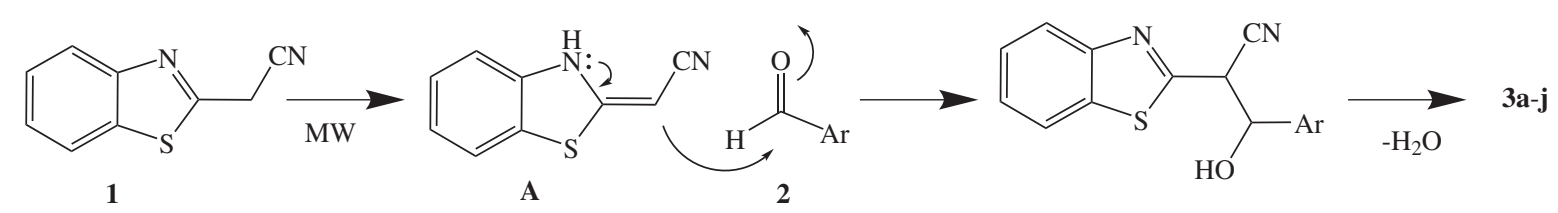

Scheme 2. Plausible formation of Knoevenagel adducts assisted by microwave irradiation.

versus the average of the other protons in the molecule with net charge $0.24834 \mathrm{e}$ and valence of 0.750091 . The latter fact is evidenced experimentally in the ${ }^{1} \mathrm{H}$ NMR spectrum for this compound, where the proton $\mathrm{H} 3$ is observed at very low field $\delta 8.25 \mathrm{ppm}$, because (as presented in this NPA analysis) it is very deshielded compared to the other protons. In the case of NPA analysis for conformer 4', all the charges on the protons are in an average; this means that the possibility of hydrogen bonds is low. As a proof of this fact, $\mathrm{H} 3$ for this molecule has a net charge $0.24035 \mathrm{e}$ and a valence of 0.73710; average values for a proton gave no such address as the deshielding effect, so that its stability can be attributed more to his $E$-type configuration about the double bond.

It seems reasonable to think that microwave irradiation promotes the Knoevenagel reactions through the methylene active component of 2-(benzo[d]thiazol-2-yl)acetonitrile $\mathbf{1}$. Under the optimized reaction conditions, $\mathbf{1}$ is likely to be converted to compound $\mathbf{A}$ containing double bond susceptible to the addition of electrophile $\mathbf{2}$ (Scheme 2).

\section{Conclusions}

We report here an efficient synthetic route to prepare $(E)$ 2-(benzo[d]thiazol-2-yl)-3-arylacrylonitriles derivatives conducted by microwave irradiation under solvent-free conditions. These conditions allowed us to obtain the compounds in short times and acceptable yields. No relationship was found between them and the nature of the substituents of the aldehydes. Further efforts to expand the scope of the chemistry on these compounds are ongoing subjects in our laboratory.

\section{Experimental}

\section{Equipments}

Microwave experiments were carried out using a focused microwave reactor (CEM Discover TM). Melting points were determined in a Buchi melting point apparatus and are reported uncorrected. The ${ }^{1} \mathrm{H}$ and ${ }^{13} \mathrm{C}$ NMR spectrum were measured at RT on a Bruker Avance 400 spectrometer operating at 400 and $100 \mathrm{MHz}$, respectively, and using $\mathrm{CDCl}_{3}$ as solvent and tetramethylsilane as internal standard. The mass-spectra were scanned on a Hewlett Packard HP Engine-5989 spectrometer (equipped with a direct inlet probe) which was operating at $70 \mathrm{eV}$. The elemental analyses have been obtained using a LECO CHNS-900 and Thermo Finnigan FlashEA1112 CHNS-O elemental analyzers. Thin layer chromatography was carried out using Merck $0.2 \mathrm{~mm}$ silica gel $60 \mathrm{~F}_{254}$ aluminum chromatographic plates.

All theoretical calculations were carried out at the DFT approach using the Gaussian 03 program review C.02. ${ }^{28}$ The DFT calculations were determined using three parameters exchange functional of Becke B3LYP and a set of orbital 3-21G basis. The net atomic charges were calculated using NPA analysis included in the algorithm natural bonding orbital (NBO) proposed by Weinhold and co-workers. ${ }^{29,30}$

\section{Reagents}

The reagents (2-(benzo[ $d]$ thiazol-2-yl)acetonitrile and aromatic aldehydes) and solvents used, such as, ethanol, ethyl acetate, hexane and DMF, were acquired commercially.

Synthesis

General procedure for the preparation of the (E)-2(benzo[d]thiazol-2-yl)-3-arylacrylonitriles $3 a-j$

A mixture of 2-(benzo[d]thiazol-2-yl)acetonitrile $\mathbf{1}$ and aromatic aldehydes $\mathbf{2}$ with electrondonating/electronwithdrawing groups $(1 \mathrm{mmol})$ were subjected to microwave irradiation (maximum power $150 \mathrm{~W}$ during 10-20 min under solvent-free conditions at a controlled temperature of $473 \mathrm{~K}$ and $250 \mathrm{psi}$ ) using a focused microwave reactor (CEM Discover). The solid products were isolated by simple crystallization of the reaction mixture from ethanol.

\section{(E)-2-(Benzo[d]thiazol-2-yl)-3-phenylacrylonitrile $\mathbf{3 a}$}

Yellow solid; $\mathrm{mp} 116-118^{\circ} \mathrm{C}, 70 \%$. IR (KBr) $v_{\max } / \mathrm{cm}^{-1}$ : $2221(\mathrm{CN}), 3060\left(-\mathrm{C}=\mathrm{H}_{\text {Aromatics }}\right), 3019(=\mathrm{C}-\mathrm{H}), 1590$ $(\mathrm{C}=\mathrm{C})$. MS (70eV) $\mathrm{m} / \mathrm{z}(\%): 263\left(9, \mathrm{M}^{+}+1\right), 262\left(32, \mathrm{M}^{+}\right)$, 261(100), 236(13). ${ }^{1} \mathrm{H}$ NMR (400 MHz, $\mathrm{CDCl}_{3}$ ) $\delta$ ppm: $7.43\left(\mathrm{t}, 1 \mathrm{H}, J 8.28 \mathrm{~Hz}, \mathrm{H}_{p}\right), 7.50-7.56$ (m, 4H, H, phenyl), $7.91(\mathrm{~d}, 1 \mathrm{H}, J 8.03 \mathrm{~Hz}, \mathrm{H} 7$ '), $8.01-8.04$ (m, 2H, H5' and 
H6'), 8.08 (d, 1H, J 7.76 Hz, H4'), 8.25 (s, 1H, CH). ${ }^{13} \mathrm{C}$ NMR (100 MHz, $\left.\mathrm{CDCl}_{3}\right) \delta$ ppm: 105.7 (C2), 116.4 (CN), 121.7 (C4'), 123.7 (C7'), 126.0 (C6'), $127.0\left(\mathrm{C}_{p}\right)$, $129.3\left(\mathrm{C}_{m}\right), 130.4\left(\mathrm{C}_{o}\right), 132.2\left(\mathrm{C}_{i}\right), 135.0\left(\mathrm{C}^{\prime}\right.$ 'a $), 147.0$ $(\mathrm{CH}), 162.7$ (C2'). Anal. calc. for $\mathrm{C}_{16} \mathrm{H}_{10} \mathrm{~N}_{2} \mathrm{~S}: \mathrm{C} 73.26 ; \mathrm{H}$ 3.84; N 10.68; found: C 73.16; H 3.83; N 10.65.

\section{(E)-2-(Benzo[d]thiazol-2-yl)-3-p-tolylacrylonitrile $3 \boldsymbol{b}$}

Yellow solid; mp $140-142{ }^{\circ} \mathrm{C}, 70 \%$. IR (KBr) $v_{\max } / \mathrm{cm}^{-1}$ : $2223(\mathrm{CN}), 3018\left(-\mathrm{C}=\mathrm{H}_{\text {Aromatics }}\right), 2855(=\mathrm{C}-\mathrm{H}), 1589$ $(\mathrm{C}=\mathrm{C})$. MS (70 eV) $\mathrm{m} / \mathrm{z}(\%): 277\left(10, \mathrm{M}^{+}+1\right), 276\left(40, \mathrm{M}^{+}\right)$, 275(100). ${ }^{1} \mathrm{H} \mathrm{NMR}\left(400 \mathrm{MHz}, \mathrm{CDCl}_{3}\right) \delta$ ppm: 2.43 (s, 3H, $\left.\mathrm{CH}_{3}\right) 7.31\left(\mathrm{~d}, 2 \mathrm{H}, J 8.28, \mathrm{H}_{m}\right), 7.41(\mathrm{t}, 1 \mathrm{H}, J 8.29 \mathrm{~Hz}, \mathrm{H} 5$ '), 7.51 (t, 1H, J 8.28 Hz, H6'), 7.89 (d, 1H, J $8.03 \mathrm{~Hz}, \mathrm{H} 4$ '), 7.93 (d, 2H, J $8.28 \mathrm{~Hz}, \mathrm{H}_{o}$ ), 8.07 (d, 1H, J 8.28 Hz, H7'), $8.21(\mathrm{~s}, 1 \mathrm{H}, \mathrm{CH})) .{ }^{13} \mathrm{C}$ NMR $\left(100 \mathrm{MHz}, \mathrm{CDCl}_{3}\right) \delta \mathrm{ppm}$ : $21.8\left(\mathrm{CH}_{3}\right), 104.4(\mathrm{C} 2), 116.7(\mathrm{CN}), 121.6\left(\mathrm{C}^{\prime}\right), 123.5$ (C7'), 125.8 (C5'), 126.8 (C6'), $129.7\left(\mathrm{C}_{i}\right), 130.0\left(\mathrm{C}_{m}\right)$, 130.5 (C $\left.\mathrm{C}_{o}\right), 134.9$ (C7'a), 140.1 (C3'a), $143.4\left(\mathrm{C}_{p}\right), 146.9$ $(\mathrm{CH}), 163.0\left(\mathrm{C} 2^{\prime}\right)$. Anal. calc. for $\mathrm{C}_{17} \mathrm{H}_{12} \mathrm{~N}_{2} \mathrm{~S}: \mathrm{C} 73.88 ; \mathrm{H}$ 4.38; N 10.14; found: C 73.80; H 4.36; N 10.17 .

\section{(E)-2-(Benzo[d]thiazol-2-yl)-3-(4-nitrophenyl)} acrylonitrile $3 c$

Yellow solid; $\mathrm{mp} 170-172{ }^{\circ} \mathrm{C}, 50 \%$. IR $(\mathrm{KBr}) v_{\max } / \mathrm{cm}^{-1}$ : $2225(\mathrm{CN}), 3031(-\mathrm{C}=\mathrm{H}) 2853(=\mathrm{C}-\mathrm{H}), 1522(\mathrm{C}=\mathrm{C}) . \mathrm{MS}$ $(70 \mathrm{eV}) \mathrm{m} / \mathrm{z}(\%): 308\left(11, \mathrm{M}^{+}+1\right), 307\left(46, \mathrm{M}^{+}\right), 306(82)$, 260(100), 261(38). ${ }^{1} \mathrm{H}$ NMR (400 MHz, $\left.\mathrm{CDCl}_{3}\right) \delta$ ppm 7.46 (t, 1H, J 7.00 Hz, H5'), 7.55 (t, 1H, J 7.00 Hz, H6'), 7.77 $\left(\mathrm{d}, 2 \mathrm{H}, J 8.53 \mathrm{~Hz}, \mathrm{H}_{m}\right) 7.93\left(\mathrm{~d}, 1 \mathrm{H}, J 8.30 \mathrm{~Hz}, \mathrm{H} 4^{\prime}\right), 8.10(\mathrm{~d}$, $\left.3 \mathrm{H}, J 8.78 \mathrm{~Hz}, \mathrm{H}_{o}, \mathrm{H} 7^{\prime}\right), 8.29$ (s, 1H, CH). ${ }^{13} \mathrm{C}$ NMR (100 $\left.\mathrm{MHz}, \mathrm{CDCl}_{3}\right) \delta$ ppm: $108.5(\mathrm{C} 2), 116.2(\mathrm{CN}), 122.1\left(\mathrm{C}^{\prime}\right)$, 124.1 (C7'), $125.2\left(\mathrm{C}_{i}\right), 126.5$ (C5'), 126.7 (C6'), 127.5 $\left(\mathrm{C}_{m}\right), 130.6\left(\mathrm{C}_{o}\right), 133.3,133.7$ (C7'a), 135.5 (C3'a), 135.9 $\left(\mathrm{C}_{p}\right), 144.9(\mathrm{CH}), 153.9\left(\mathrm{C} 2^{\prime}\right)$. Anal. calc. for $\mathrm{C}_{16} \mathrm{H}_{9} \mathrm{~N}_{3} \mathrm{O}_{2} \mathrm{~S}$ : C 62.53; H 2.95; N 13.67; found: C 62.54; H 2.92; N 13.72.

\section{(E)-2-(Benzo[d]thiazol-2-yl)-3-(4-chlorophenyl) acrylonitrile $\mathbf{3 d}$}

Yellow solid; mp $144-148^{\circ} \mathrm{C}, 75 \%$. IR $(\mathrm{KBr}) v_{\max } / \mathrm{cm}^{-1}$ : $2219(\mathrm{CN}), 3060\left(-\mathrm{C}=\mathrm{H}_{\text {Aromatics }}\right), 2855(=\mathrm{C}-\mathrm{H}), 1584$ $(\mathrm{C}=\mathrm{C}) . \mathrm{MS}(70 \mathrm{eV}) \mathrm{m} / \mathrm{z}(\%): 298\left(15, \mathrm{M}^{+}+2\right), 296(42$, $\mathrm{M}^{+}$), 295(100), 270(17), 261(11). ${ }^{1} \mathrm{H}$ NMR (400 MHz, DMSO- $\left.d_{6}\right) \delta$ ppm 7.51 (t, $1 \mathrm{H}, J 7.28 \mathrm{~Hz}, \mathrm{H} 5$ '), $7.58(\mathrm{t}, 1 \mathrm{H}$, $J 7.53 \mathrm{~Hz}, \mathrm{H6}$ ') $7.66\left(\mathrm{~d}, 2 \mathrm{H}, J 8.79 \mathrm{~Hz}, \mathrm{H}_{m}, 8.07(\mathrm{~d}, 1 \mathrm{H}\right.$, $\left.J 7.53 \mathrm{~Hz}, \mathrm{H} 4^{\prime}\right), 8.09$ (d, 2H, J $\left.9.03 \mathrm{~Hz}, \mathrm{H}_{o}\right), 8.17$ (d, 2H, $J 8.03 \mathrm{~Hz}, \mathrm{H} 7$ '), 8.40 (s, 1H, CH). ${ }^{13} \mathrm{C}$ NMR $(100 \mathrm{MHz}$, $\left.\mathrm{CDCl}_{3}\right) \delta$ ppm: $106.1(\mathrm{C} 2), 115.8(\mathrm{CN}), 122.4\left(\mathrm{C}^{\prime}\right), 123.1$ (C7'), 126.3 (C5'), 127.1 (C6'), $129.3\left(\mathrm{C}_{m}\right), 131.1\left(\mathrm{C}_{i}\right)$, $131.7\left(\mathrm{C}_{\circ}\right), 134.3$ (C7'a), 136.8 (C3'a), 146.7 (CH), 152.8 $\left(\mathrm{C}_{p}\right), 162.8\left(\mathrm{C}^{\prime}\right)$.
(E)-2-(Benzo[d]thiazol-2-yl)-3-(4-bromophenyl) acrylonitrile $3 \boldsymbol{e}$

Brown solid; mp $138-140{ }^{\circ} \mathrm{C}, 50 \%$. IR (KBr) $v_{\max } / \mathrm{cm}^{-1}$ : $2219(\mathrm{CN}), 3062\left(-\mathrm{C}=\mathrm{H}_{\text {Aromatics }}\right), 3020(=\mathrm{C}-\mathrm{H}), 1579$ $(\mathrm{C}=\mathrm{C})$. MS $(70 \mathrm{eV}) \mathrm{m} / \mathrm{z}(\%): 341\left(17, \mathrm{M}^{+}+2\right), 339\left(16, \mathrm{M}^{+}\right)$, 280(40), 279(100), 254(21). ${ }^{1} \mathrm{H} \mathrm{NMR}\left(400 \mathrm{MHz}, \mathrm{CDCl}_{3}\right) \delta$ ppm: 7.44 (t, 1H, J 7.28 Hz, H5'), 7.54 (t, $1 \mathrm{H}, J 7.03 \mathrm{~Hz}$, H6'), $7.64\left(\mathrm{~d}, 2 \mathrm{H}, J 8.53, \mathrm{H}_{m}\right), 7.87\left(\mathrm{~d}, 2 \mathrm{H}, J 8.78 \mathrm{~Hz}, \mathrm{H}_{o}\right)$, 7.91 (d, 1H, J 8.03 Hz, H4'), 8.08 (d, 1H, J 8.04 Hz, H7'), $8.18(\mathrm{~s}, 1 \mathrm{H}, \mathrm{CH}) .{ }^{13} \mathrm{C} \mathrm{NMR}\left(100 \mathrm{MHz}, \mathrm{CDCl}_{3}\right) \delta \mathrm{ppm}$ : 106.1 (C2), $116.2(\mathrm{CN}), 121.7$ (C4'), 123.7 (C7'), 126.1 (C5'), $126.9\left(\mathrm{C}_{i}\right), 127.0\left(\mathrm{C}^{\prime}\right), 131.5\left(\mathrm{C}_{m}\right), 132.6\left(\mathrm{C}_{o}\right)$, 135.0 (C7'a), $145.2(\mathrm{CH}), 153.4\left(\mathrm{C}_{p}\right), 162.3$ (C2'). Anal. calc. for $\mathrm{C}_{16} \mathrm{H}_{9} \mathrm{BrN}_{2} \mathrm{~S}$ : C 56.32; H 2.66; N 8.21; found: C $56.33 ; \mathrm{H} 2.63 ; \mathrm{N} 8.17$.

(E)-2-(Benzo[d]thiazol-2-yl)-3-(4-fluorophenyl) acrylonitrile $3 f$

Brown solid; $\mathrm{mp} 150-152^{\circ} \mathrm{C}, 50 \%$. IR ( KBr) $v_{\max } / \mathrm{cm}^{-1}$ : $2224(\mathrm{CN}), 3052\left(-\mathrm{C}=\mathrm{H}_{\text {Aromatics }}\right), 1588(\mathrm{C}=\mathrm{C}) . \mathrm{MS}$ $(70 \mathrm{eV}) \mathrm{m} / z$ (\%): 281(10, $\left.\mathrm{M}^{+}+1\right), 280\left(39, \mathrm{M}^{+}\right), 279(100)$, 254(22). ${ }^{1} \mathrm{H}$ NMR (400 MHz, $\left.\mathrm{CDCl}_{3}\right) \delta$ ppm: $7.19(\mathrm{t}, 2 \mathrm{H}$, $\left.J 8.53 \mathrm{~Hz}, \mathrm{H}_{m}\right), 7.43(\mathrm{t}, 1 \mathrm{H}, J 7.02 \mathrm{~Hz}, \mathrm{H} 5$ ') $7.52(\mathrm{t}, 1 \mathrm{H}$, $J 7.02 \mathrm{~Hz}, \mathrm{H}^{\prime}$ '), 7.90 (d, 1H, J 8.28 Hz, H4'), 8.02-8.07 (m, 3H, Ho, $\mathrm{H}^{\prime}$ '), 8.20 (s, 1H, CH). ${ }^{13} \mathrm{C}$ NMR $(100 \mathrm{MHz}$, $\left.\mathrm{CDCl}_{3}\right) \delta$ ppm: $105.2(\mathrm{C} 2), 116.4(\mathrm{CN}), 116.7\left(\mathrm{C}_{m}\right)$, 121.7 (C4'), 123.6 (C7'), 126.0 (C5'), 127.0 (C6'), 128.7 $\left(\mathrm{C}_{i}\right), 132.7\left(\mathrm{C}_{o}\right), 134.9\left(\mathrm{C} 7\right.$ 'a), $145.3(\mathrm{CH}), 153.6\left(\mathrm{C}_{p}\right)$, 162.5 (C2').

(E)-2-(Benzo[d]thiazol-2-yl)-3-(4-(trifluoromethyl)phenyl) acrylonitrile $3 g$

Beige solid; mp 122-124 ${ }^{\circ} \mathrm{C}, 75 \%$. IR (KBr) $v_{\max } / \mathrm{cm}^{-1}$ : $2229(\mathrm{CN}), 3066\left(-\mathrm{C}=\mathrm{H}_{\text {Aromatics }}\right), 2942(=\mathrm{C}-\mathrm{H}), 1476$ $(\mathrm{C}=\mathrm{C})$. MS $(70 \mathrm{eV}) \mathrm{m} / z(\%): 331\left(10, \mathrm{M}^{+}+1\right), 330(38$, $\mathrm{M}^{+}$), 329(100), 304(13), 261(11). ${ }^{1} \mathrm{H}$ NMR (400 MHz, $\left.\mathrm{CDCl}_{3}\right) \delta$ ppm: $7.48(\mathrm{t}, 1 \mathrm{H}, J 7.03 \mathrm{~Hz}, \mathrm{H} 5$ '), $7.56(\mathrm{t}, 1 \mathrm{H}$, $J 7.03$ Hz, H6'), 7.93 (d, 1H, J 7.78 Hz, H4') 8.10 (d, 1H, $J 8.04 \mathrm{~Hz}, \mathrm{H} 7$ '), 8.14 (d, 2H, $J 8.79 \mathrm{~Hz} \mathrm{H}$ ), 8.30 (s, 1H, $\mathrm{CH}), 8.34\left(\mathrm{~d}, 2 \mathrm{H}, J 8.78 \mathrm{~Hz}, \mathrm{H}_{m}\right) \cdot{ }^{13} \mathrm{C}$ NMR $(100 \mathrm{MHz}$, $\left.\mathrm{CDCl}_{3}\right) \delta$ ppm: 109.5 (C2), 115.6 (CN), 121.80 (C4'), 124.0 (C7'), 124.3 (C5'), 126.6 (C6'), $127.3\left(\mathrm{C}_{m}\right), 130.8\left(\mathrm{C}_{o}\right)$, 135.3 (C7'a), 138.0 (C3'a), 143.2 (CH), 161.2 (C2'). Anal. calc. for: C 61.81; H 2.75; N 8.48; found: C 61.82; H 2.71; N 8.49 .

(E)-2-(Benzo[d]thiazol-2-yl)-3-(4-methoxyphenyl) acrylonitrile $\mathbf{3 h}$

Yellow solid; mp $130{ }^{\circ} \mathrm{C}$ (decomposition); 50\%. IR $(\mathrm{KBr}) v_{\max } / \mathrm{cm}^{-1}: 2219(\mathrm{CN}), 3007\left(-\mathrm{C}=\mathrm{H}_{\text {Aromatics }}\right), 2833$ $(=\mathrm{C}-\mathrm{H}), 1589(\mathrm{C}=\mathrm{C}) . \mathrm{MS}(70 \mathrm{eV}) \mathrm{m} / \mathrm{z}(\%): 293\left(13, \mathrm{M}^{+}+1\right)$, 
292(58, M+), 291(100), 277(11), 266(27), 261(13), 248(41). ${ }^{1} \mathrm{H}$ NMR (400 MHz, $\left.\mathrm{CDCl}_{3}\right) \delta$ ppm: 3.87 (s, $3 \mathrm{H}, \mathrm{OCH}_{3}$ ), 6.99 (d, 2H, J $8.79 \mathrm{~Hz}, \mathrm{H}_{m}$ ), 7.39 (t, $1 \mathrm{H}, J 8.03 \mathrm{~Hz}, \mathrm{H} 5$ '), 7.49 (t, 1H, J $8.03 \mathrm{~Hz}, \mathrm{H6}$ '), 7.87 (d, 1H, J $8.03 \mathrm{~Hz}, \mathrm{H} 4$ '), 8.00 (d, 2H, J $\left.8.79 \mathrm{~Hz}, \mathrm{H}_{o}\right), 8.02$ (d, 1H, J $8.28 \mathrm{~Hz}, \mathrm{H} 7$ '), 8.14 (s, $1 \mathrm{H}, \mathrm{CH}) .{ }^{13} \mathrm{C}$ NMR $\left(100 \mathrm{MHz}, \mathrm{CDCl}_{3}\right) \delta \mathrm{ppm}: 55.8$ $\left(\mathrm{OCH}_{3}\right), 102.6(\mathrm{C} 2), 115.0\left(\mathrm{C}_{m}\right), 117.3(\mathrm{CN}), 121.9\left(\mathrm{C}^{\prime}{ }^{\prime}\right)$, 123.6 (C7'), $125.5\left(\mathrm{C}_{i}\right), 126.0$ (C5'), 127.1 (C6'), 133.0 $\left(\mathrm{C}_{o}\right), 135.1$ (C7'a), $146.7(\mathrm{CH}), 153.9\left(\mathrm{C}_{p}\right), 163.2(\mathrm{C} 2$ '). Anal. calc. for $\mathrm{C}_{17} \mathrm{H}_{12} \mathrm{~N}_{2} \mathrm{OS}$ : C 69.84; $\mathrm{H} 4.14 ; \mathrm{N} 9.58$; found: C 69.86; H 4.15; N 9.55.

(E)-2-(Benzo[d]thiazol-2-yl)-3-(3,4,5-trimethoxyphenyl) acrylonitrile $3 \boldsymbol{i}$

Yellow solid; mp 140-142 ${ }^{\circ} \mathrm{C}, 55 \%$. IR (KBr) $v_{\max } / \mathrm{cm}^{-1}$ : $2210(\mathrm{CN}), 3055\left(-\mathrm{C}=\mathrm{H}_{\text {Aromatics }}\right), 2838(=\mathrm{C}-\mathrm{H}), 1573$ $(\mathrm{C}=\mathrm{C})$. MS (70 eV) $\mathrm{m} / \mathrm{z}(\%): 353\left(14, \mathrm{M}^{+}+1\right), 352\left(53, \mathrm{M}^{+}\right)$, 357(37), 151(100). ${ }^{1} \mathrm{H}$ NMR (400 MHz, $\mathrm{CDCl}_{3}$ ) $\delta$ ppm: $3.93\left(\mathrm{~s}, 6 \mathrm{H}, \mathrm{OCH}_{3}-\mathrm{C}_{m}\right.$ ), 3.95 (s, $3 \mathrm{H}, \mathrm{OCH}_{3}-\mathrm{C}_{p}$ ), 7.31 (s, $\left.2 \mathrm{H}, \mathrm{H}_{o}\right), 7.41$ (t, 1H, J 7.03 Hz, H5'), 7.51 (t, 1H, J 7.03 Hz, H6'), 7.89 (d, 1H, J 7.03 Hz, H4'), 8.05 (d, 1H, J 7.28 Hz, H7'), 8.15 (s, 1H, CH). ${ }^{13} \mathrm{C}$ NMR (100 $\left.\mathrm{MHz}, \mathrm{CDCl}_{3}\right)$ $\delta$ ppm: $56.3\left(\mathrm{OCH}_{3}-\mathrm{C}_{m}\right), 61.1\left(\mathrm{OCH}_{3}-\mathrm{C}_{p}\right), 104.0(\mathrm{C} 2)$, $107.9\left(\mathrm{C}_{o}\right), 116.9(\mathrm{CN}), 121.6\left(\mathrm{C}^{\prime}\right), 123.4\left(\mathrm{C}^{\prime}\right.$ '), 125.9 (C5'), 126.9 (6'), 127.5 (C $\left.\mathrm{C}_{i}\right), 134.9$ (C7'a), 141.9 (C3'a), $146.7(\mathrm{CH}), 153.3\left(\mathrm{C}_{m}\right), 153.6\left(\mathrm{C}_{p}\right), 162.9\left(\mathrm{C} 2^{\prime}\right)$. Anal. calc. for $\mathrm{C}_{19} \mathrm{H}_{16} \mathrm{~N}_{2} \mathrm{O}_{3} \mathrm{~S}$ : C 64.76; H 4.58; N 7.95; found: C 64.74; H 4.59; N 7.91.

(E)-3-(Benzo[d][1,3]dioxol-6-yl)-2-(benzo[d]thiazol-2-yl) acrylonitrile $\mathbf{3 j}$

Yellow solid; mp $192-194^{\circ} \mathrm{C}, 50 \%$. IR (KBr) $v_{\max } / \mathrm{cm}^{-1}$ : $2211(\mathrm{CN}), 3065\left(-\mathrm{C}=\mathrm{H}_{\text {Aromatics }}\right), 2918(=\mathrm{C}-\mathrm{H}), 1570$ $(\mathrm{C}=\mathrm{C})$. MS $(70 \mathrm{eV}) \mathrm{m} / \mathrm{z}(\%): 307\left(14, \mathrm{M}^{+}+1\right), 306(56$, $\mathrm{M}^{+}$), 305(100), 280(16), 248(16). ${ }^{1} \mathrm{H}$ NMR (400 MHz, $\left.\mathrm{CDCl}_{3}\right) \delta$ ppm: $6.11\left(\mathrm{~s}, 2 \mathrm{H}, \mathrm{CH}_{2}\right), 6.95(\mathrm{~d}, 1 \mathrm{H}, J 8.03 \mathrm{~Hz}$, H5-aryl), 7.43 (t, 1H, J $8.28 \mathrm{~Hz}, \mathrm{H} 5$ ') 7.47 (d, 1H, $J 8.28 \mathrm{~Hz}, \mathrm{H} 6$-aryl), 7.52 (t, 1H, J 8.28 Hz, H6'), 7.71 (s, $1 \mathrm{H}, \mathrm{H} 2$-aryl), 7.93 (d, 1H, J $\left.8.53 \mathrm{~Hz}, \mathrm{H} 4^{\prime}\right), 8.03$ (d, 1H, $J 8.03 \mathrm{~Hz}, \mathrm{H} 7$ '), 8.12 (s, 1H, CH). ${ }^{13} \mathrm{C}$ NMR $(100 \mathrm{MHz}$, $\left.\mathrm{CDCl}_{3}\right) \delta$ ppm: $101.5\left(\mathrm{CH}_{2}\right), 102.2(\mathrm{C} 2), 107.6(\mathrm{CH}-a r y l)$, 108.2 (CH-aryl), 115.9 (CN), 120.9 (C4'), 122.6 (C7'), 125.1 (C5'), 126.1 (C6'), 127.6 (CH-aryl), $134.0\left(\mathrm{C}_{q}\right)$, $146.0(\mathrm{CH}), 147.9\left(\mathrm{C}_{q}\right), 150.7\left(\mathrm{C}_{q}\right), 152.8\left(\mathrm{C}_{q}\right), 162.4$ (C2'). Anal. calc. for $\mathrm{C}_{16} \mathrm{H}_{9} \mathrm{~N}_{3} \mathrm{O}_{2} \mathrm{~S}$ : C 62.53; H 2.95; N 13.67; found: C 62.54; H 2.92; N 13.62.

\section{Supplementary Information}

Supplementary information (Figures S1-S20) is available free of charge at http://jbcs.sbq.org.br as PDF file.

\section{Acknowledgments}

The authors thank Universidad del Atlántico, Universidad del Valle (Colombia) and COLCIENCIAS for financial support.

\section{References}

1. Dawood, K. M.; Elwan, N. M.; Farahat, A. A.; Abdel-Wahab, B. F.; J. Heterocycl. Chem. 2010, 47, 243.

2. Quiroga, J.; Cruz, S.; Insuasty, B.; Abonía, R.; Nogueras, M.; Sánchez, A.; Cobo, J.; Low, J. N.; J. Heterocycl. Chem. 2001, 38,53 .

3. Quiroga, J.; Cruz, S.; Insuasty, B.; Abonía, R.; Heterocycl. Commun. 2000, 6, 275.

4. Mavrava, A. T.; Anchina, K. K.; Vuchev, D. I.; Tesnove, J. A.; Kondeva, M. S.; Miteka, K. M.; Bioorg. Med. Chem. 2005, 13, 5550.

5. Yildiz-Oren, I.; Yalcin, I.; Aki-Sener, E.; Ukarturk, N.; Eur. J. Med. Chem. 2004, 39, 291.

6. Carta, A.; Palomba, M.; Boatto, G.; Busonera, B.; Murreddu, M.; Loddo, R.; FARMACO 2004, 59, 637.

7. Quiroga, J.; Cobo, D.; Insuasty, I.; Abonía, R.; Nogueras, M.; Cobo, J.; Vásquez, Y.; Gupta, M.; Derita, M.; Zacchino, S.; Arch. Pharm. 2007, 340, 603.

8. Zhao, K.; Xu, X-P.; Zhu, S-L.; Shi, D-Q.; Zhang, Y.; Ji, S-J.; Synthesis 2009, 16, 2697.

9. Thirumurugan, P.; Perumal, P. T.; Tetrahedron 2009, 65, 7620.

10. Hranjec, M.; Pavlovic, G.; Marjanovic, M.; Kralj, M.; Karminski-Zamola, G.; Eur. J. Med. Chem. 2010, 45, 2405.

11. Refaat, H. M.; Eur. J. Med. Chem. 2010, 45, 2949.

12. Bougrin, K.; Loupy, A.; Soufiaoui, M.; J. Photochem. Photobiol., C 2005, 6, 139.

13. Quiroga, J.; Trilleras, J.; Sánchez, A.; Insuasty, B.; Abonía, R.; Nogueras, M.; Cobo, J.; Lett. Org. Chem. 2009, 6, 381.

14. Quiroga, J.; Trilleras, J.; Gálvez, J.; Insuasty, B.; Abonía, R.; Nogueras, M.; Cobo, J.; Tetrahedron Lett. 2009, 50, 6404.

15. Gálvez, J.; Quiroga, J.; Cobo, J.; Low, J.; Glidewell, C.; Acta Crystallogr., Sect. C: Cryst. Struct. Commun. 2008, 64, 385.

16. Hirata, Y.; Yada, A.; Morita, E.; Nakao, Y.; Hiyama, T.; Ohashi, M.; Ogoshi, S.; J. Am. Chem. Soc. 2010, 132, 10070.

17. Tomioka, T.; Takahashi, Y.; Vaughan, T. G.; Yanase, T.; Org. Lett. 2010, 12, 2171.

18. Fadda, A. A.; Hammouda, M.; Afsah, E. M.; Hanash, A. A.; Phosphorus, Sulfur Silicon Relat. Elem. 2010, 185, 433.

19. Fadda, A. A.; Zaki, M.; Samir, K.; Etman, H. A.; Phosphorus, Sulfur Silicon Relat. Elem. 2008, 183, 1801.

20. Dryanska, V.; Phosphorus, Sulfur, Silicon Relat. Elem. 1991, 61,325 .

21. Fathy, N. M.; Abdel Motti, F. M.; Elgemeie, G. E. H.; Arch. Pharm. 1988, 321, 509. 
22. Dryanska, V.; Sulfur Lett. 1986, 5, 47.

23. Saito, K.; Kambe, S.; Nakano, Y.; Syntheis 1983, 3, 210.

24. Lenardão, E. J.; Silva, M. S.; Mendes, S. R., De Azambuja, F.; Jacob, R. G.; Silva dos Santos, P. C.; Perin, G.; J. Braz. Chem. Soc. 2007, 18, 943.

25. Perin, G., Jacob, R. G.; Botteselle, G. V.; Kublik, E. L.; Lenardão, E. J.; Cella, R.; Silva dos Santos, P. C.; J. Braz. Chem. Soc. 2005, 16, 857.

26. Sagrera, G. J.; Seoane, G. A.; J. Braz. Chem. Soc. 2005, 16, 851.

27. Chrostowska, A.; Mai Nguyen, T. X.; Dargelos, A.; Khayar, S.; Gracia, A.; Guillemin, J-C.; J. Phys. Chem. A 2009, 113, 2387.

28. Frish, M. J.; Trucks, G. W.; Schlegel, H. B.; Scuseria, G. E.; Robb, M. A.; Cheeseman, J. R.; Vreven, T.; Montgomery, J. A.; Kudin, K. N.; Burant, J. C.; Iyengar, S. S.; Tomasi, J.; Millam, J. M.; Scalmani, G.; Barone, V.; Mennucci, B.; Cossi, M.; Rega, N.; Petersson, G. A.; Nakatsuji, H.; Hada, M.; Ehara, M.; Toyota, K.; Fukuda, R.; Hasegawa, J.; Ishida, M.; Nakajima, T.; Honda, Y.; Kitao, O.; Nakai, H.; Klene,M.; Knox,
J. E.; Li, X.; Hratchian, H. P.; Cross, J. B.; Bakken, V.; Adamo, C.; Jaramillo, J.; Gomperts, R.; Stratmann, R. E.; Yazyev, O.; Austin, A. J.; Cammi, R.; Pomelli, C.; Ochterski, J. W.; Ayala, P. Y.; Morokuma, K.; Zakrzewski, V. G.; Voth, G. A.; Salvador, P.; Dannenberg, J. J.; Dapprich, S.; Daniels, A. D.; Strain, M. C.; Farkas, O.; Malick, D. K.; Rabuck, A. D.;Raghavachari, K.; Foresman, J. B.; Ortiz, J. V.; Cui, Q.; Baboul, A. G.; Clifford, S.; Cioslowski, J.; Stefanov, B. B.; Liu, G.; Liashenko, A.; Piskorz, P.; Komaromi, I.; Martin, R. L.; Fox, D. J.; Keith, T.; Al-Laham, M. A.; Peng, C. Y.; Nanayakkara, A.; Challacombe, M.; Gill, P. M. W.; Johnson, B.; Chen, W.; Wong, M. W.; Gonzalez, C.; Pople, A.; Gaussian 03, Revision C.02; Gaussian, Inc.: Wallingford CT, 2004.

29. Reed, A. E.; Weinhold, F.; Weinstock, R. B.; J. Chem. Phys. 1985, 83, 735.

30. Reed, A. E.; Weinhold, F.; J. Chem. Phys. 1983, 78, 4066.

Submitted: March 17, 2011

Published online: September 6, 2011 


\section{Microwave-Assisted Synthesis under Solvent-Free Conditions of (E)-2-(Benzo[d]thiazol-2-yl)-3-arylacrylonitriles}

\section{Jorge E. Trilleras, ${ }^{*, a}$ Kelly J. Velasquez, ${ }^{a}$ Dency J. Pacheco, ${ }^{a}$ Jairo Quiroga ${ }^{b}$ and Alejandro Ortíz}

${ }^{a}$ Grupo de Investigación en Compuestos Heterocíclicos, Programa de Química, Facultad de Ciencias Básicas, Universidad del Atlántico, Km 7 Antigua vía Puerto Colombia, Barranquilla-Atlántico, Colombia

${ }^{b}$ Grupo de Investigación de Compuestos Heterocíclicos, Departamento de Química, Universidad del Valle, A. A 25360 Cali, Colombia
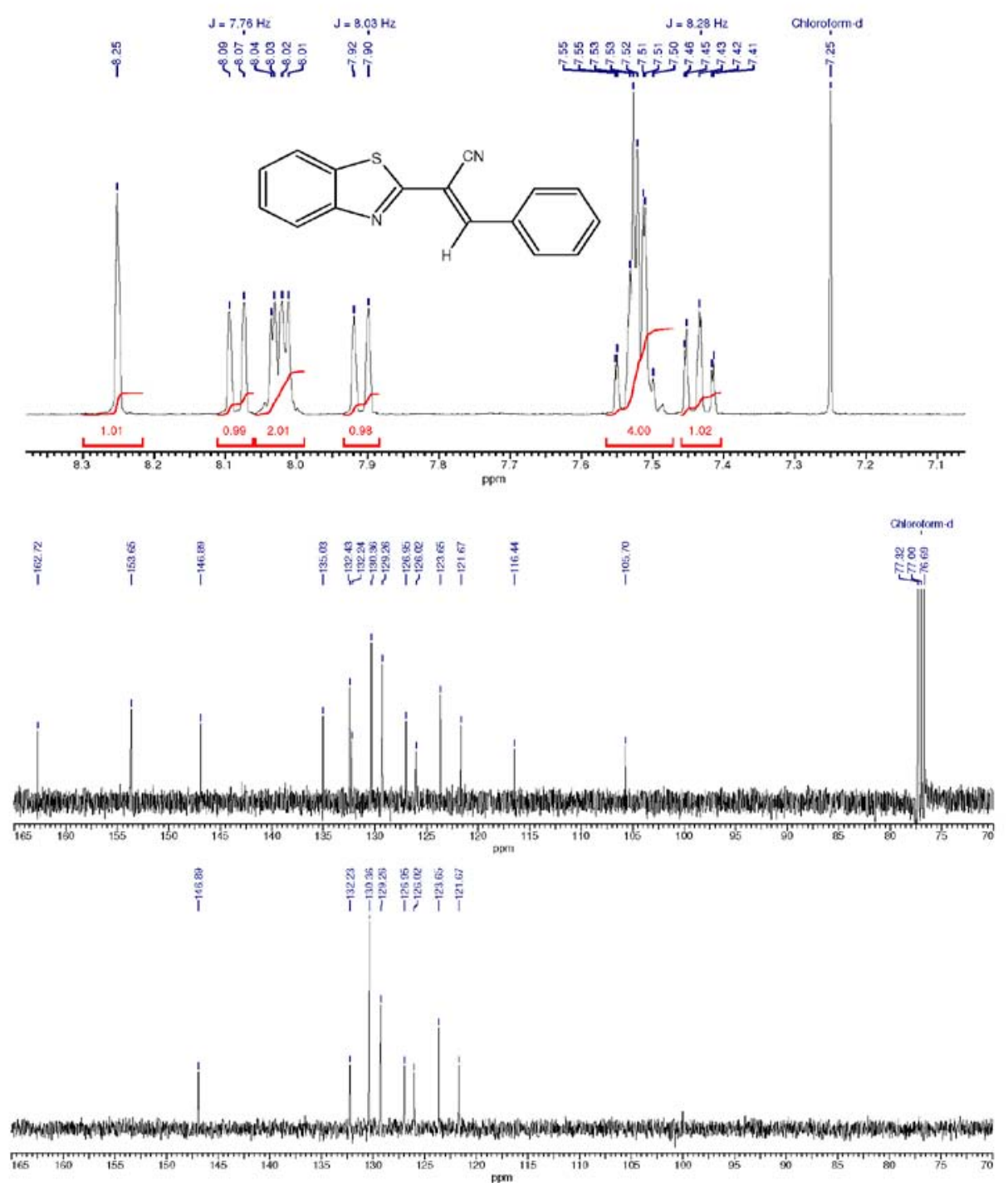

Figure S1. ${ }^{1} \mathrm{H}$ and ${ }^{13} \mathrm{C}$ and DEPT-135 NMR spectra for compound 3a.

*e-mail: jorgetrilleras@mail.uniatlantico.edu.co 


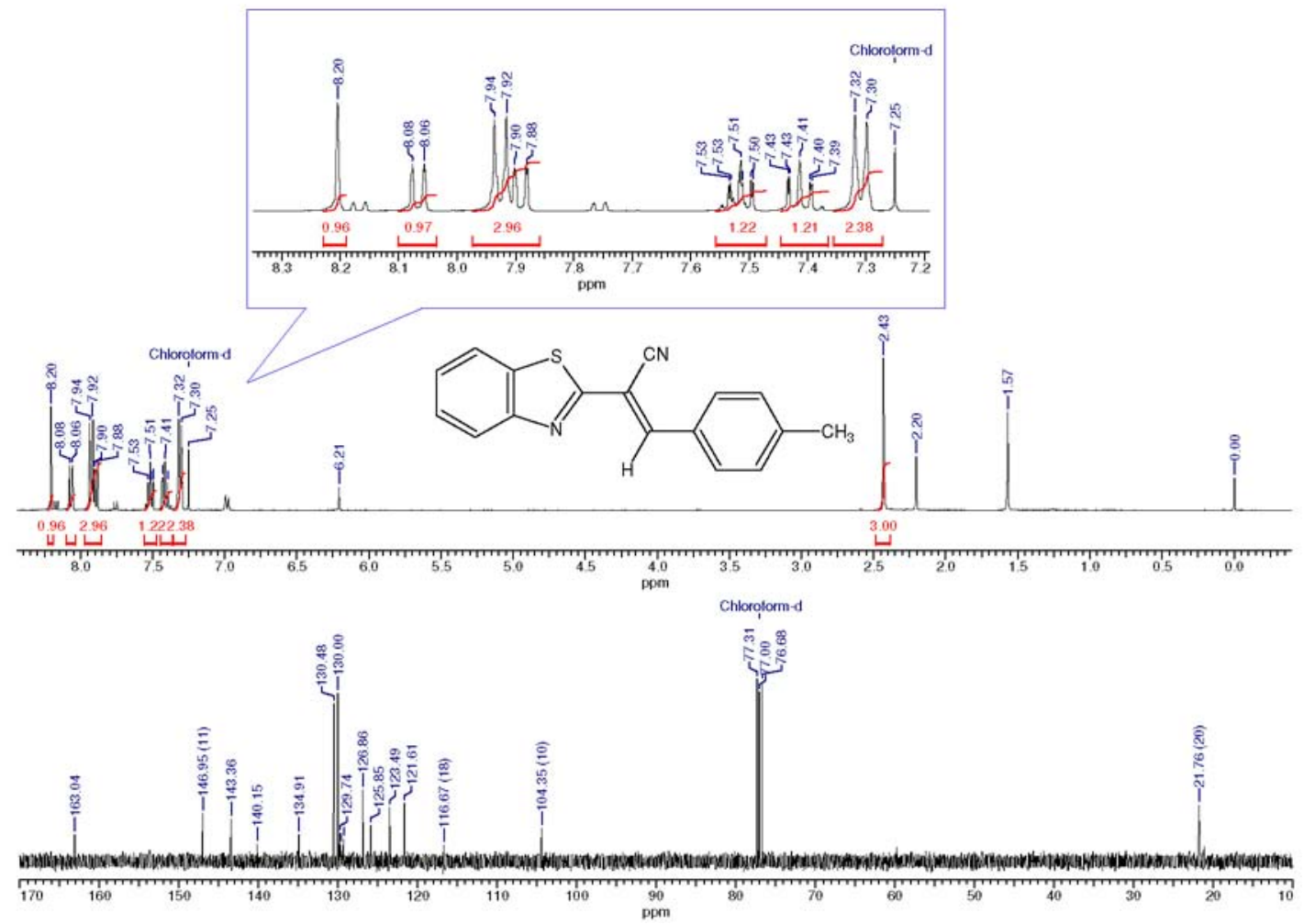

Figure S2. ${ }^{1} \mathrm{H}$ and ${ }^{13} \mathrm{C}$ NMR spectra for compound $\mathbf{3 b}$. 

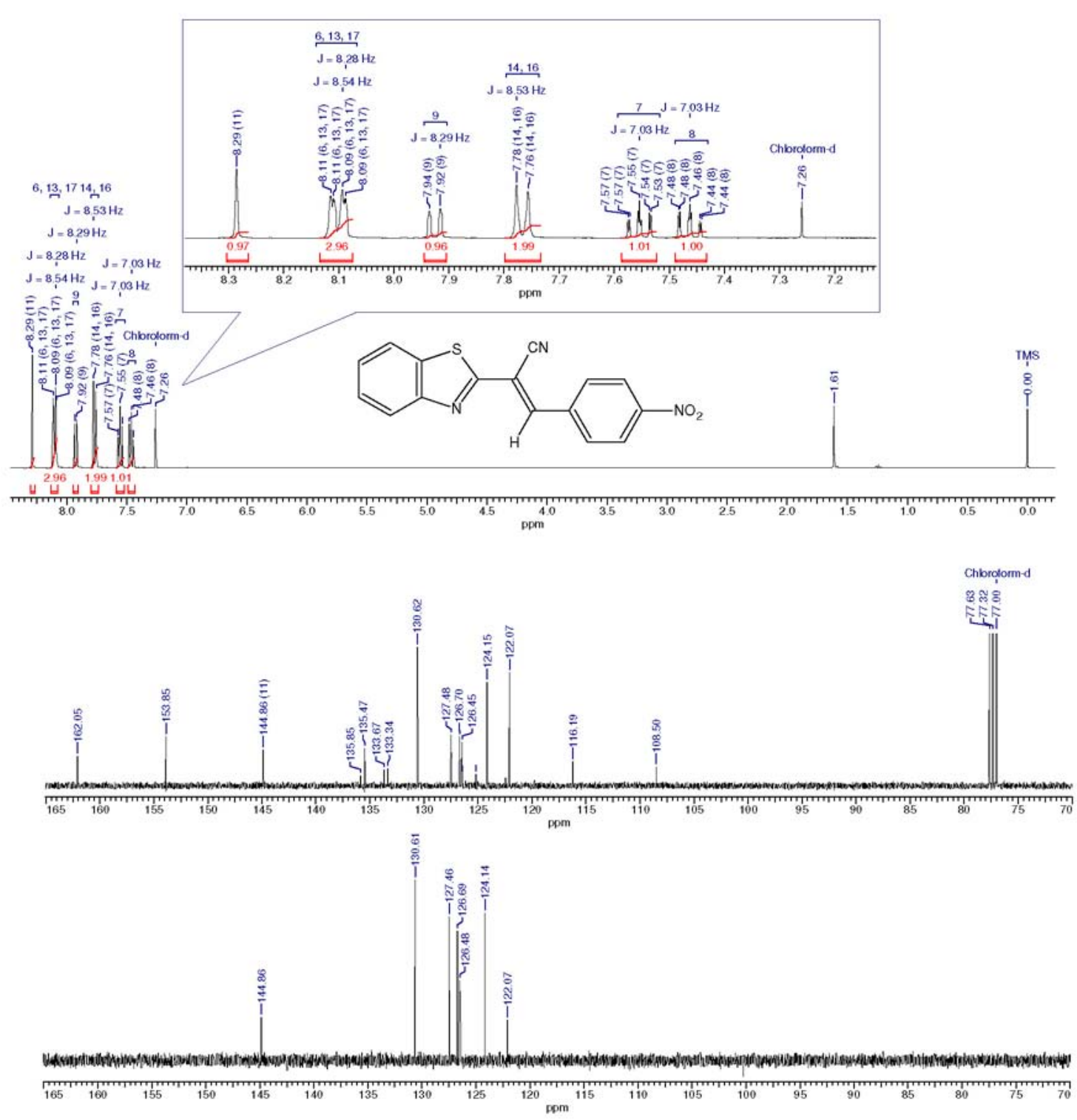

Figure S3. ${ }^{1} \mathrm{H},{ }^{13} \mathrm{C}$ and DEPT-135 NMR spectra for compound $\mathbf{3 c}$. 

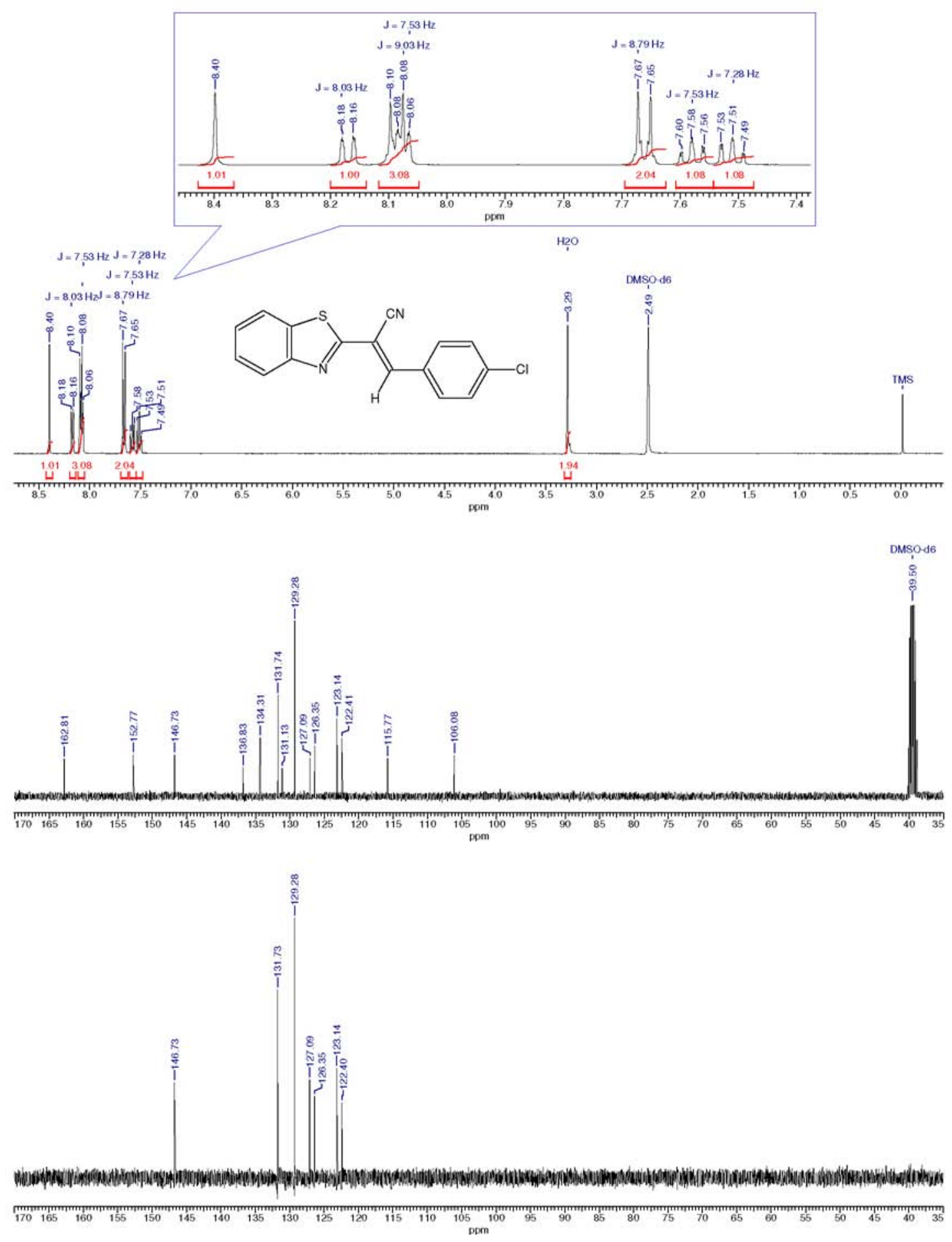

Figure S4. ${ }^{1} \mathrm{H},{ }^{13} \mathrm{C}$ and DEPT-135 spectra for compound $\mathbf{3 d}$. 


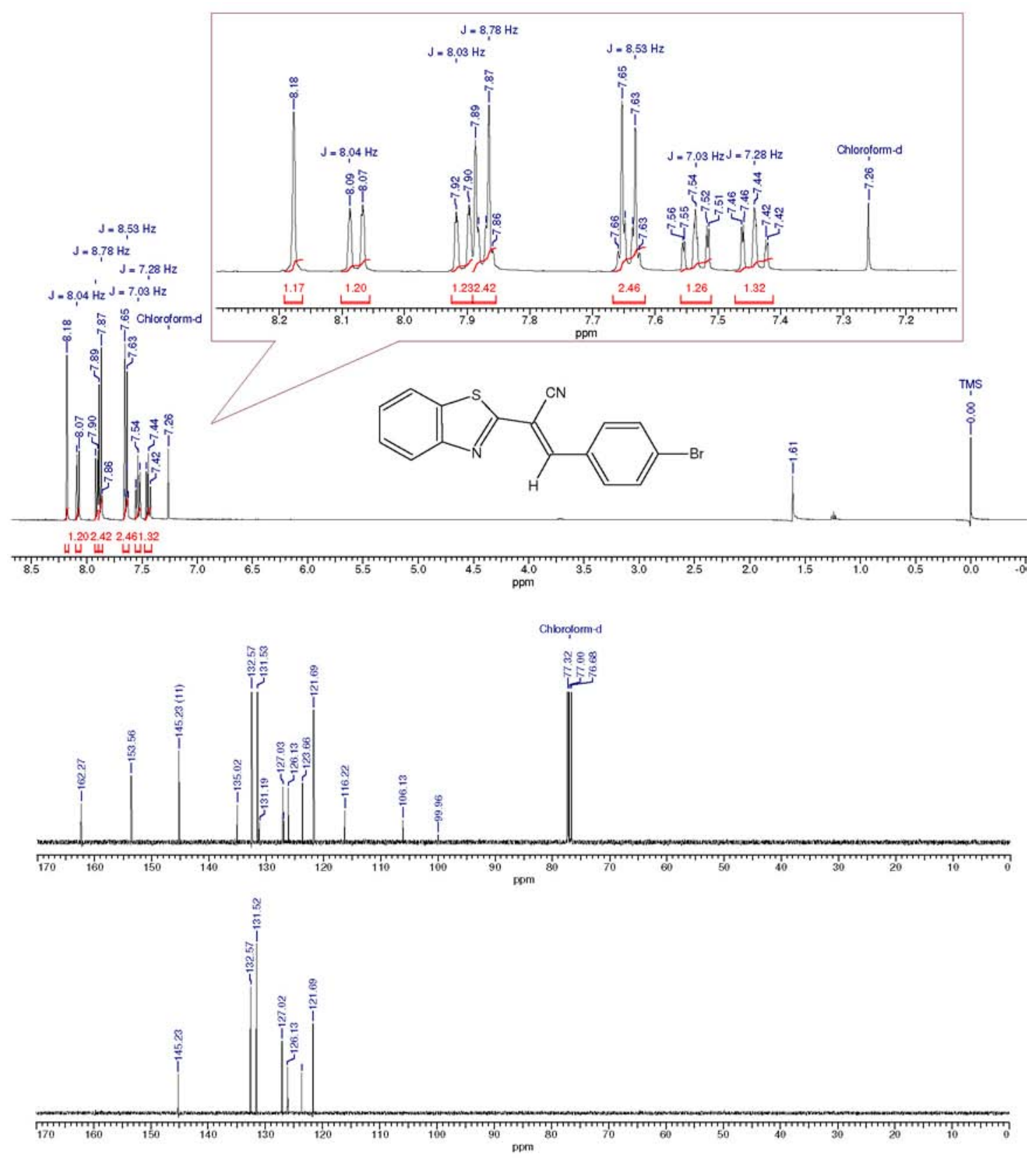

Figure S5. ${ }^{1} \mathrm{H},{ }^{13} \mathrm{C}$ and DEPT-135 spectra for compound $\mathbf{3 e}$. 

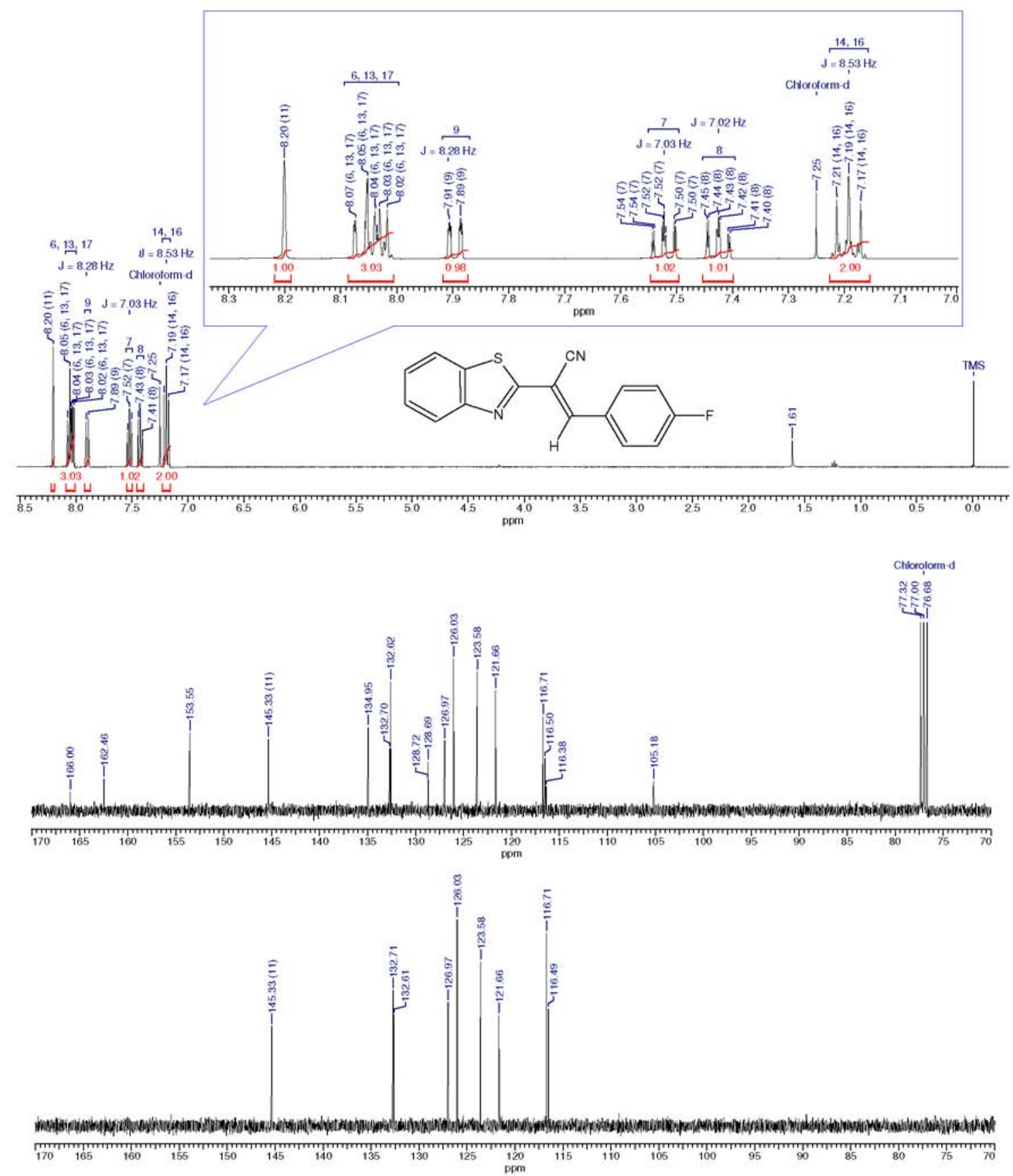

Figure S6. ${ }^{1} \mathrm{H},{ }^{13} \mathrm{C}$ and DEPT- 135 spectra for compound $\mathbf{3 f}$. 

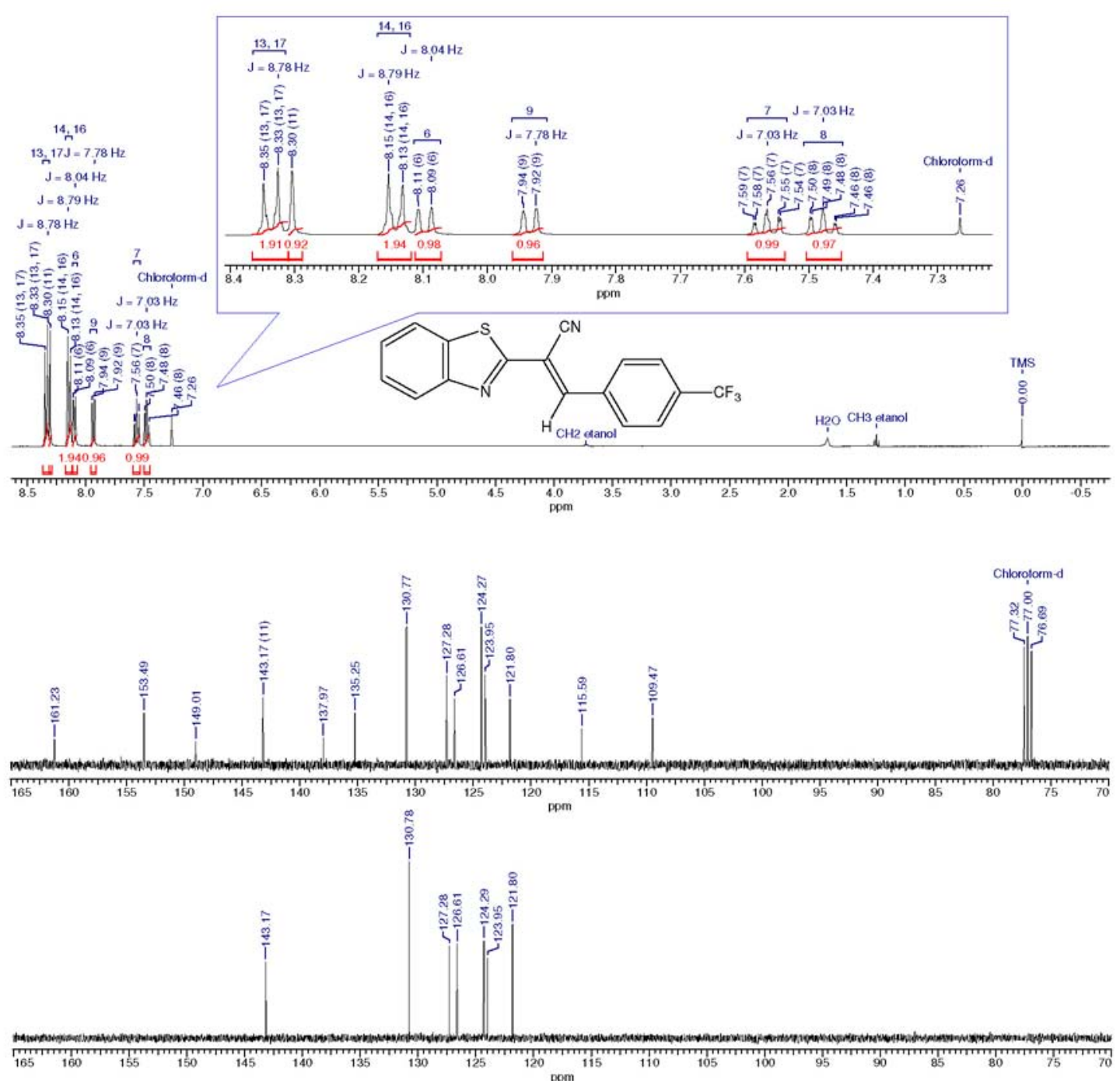

Figure S7. ${ }^{1} \mathrm{H},{ }^{13} \mathrm{C}$ and DEPT-135 spectra for compound $\mathbf{3 g}$. 

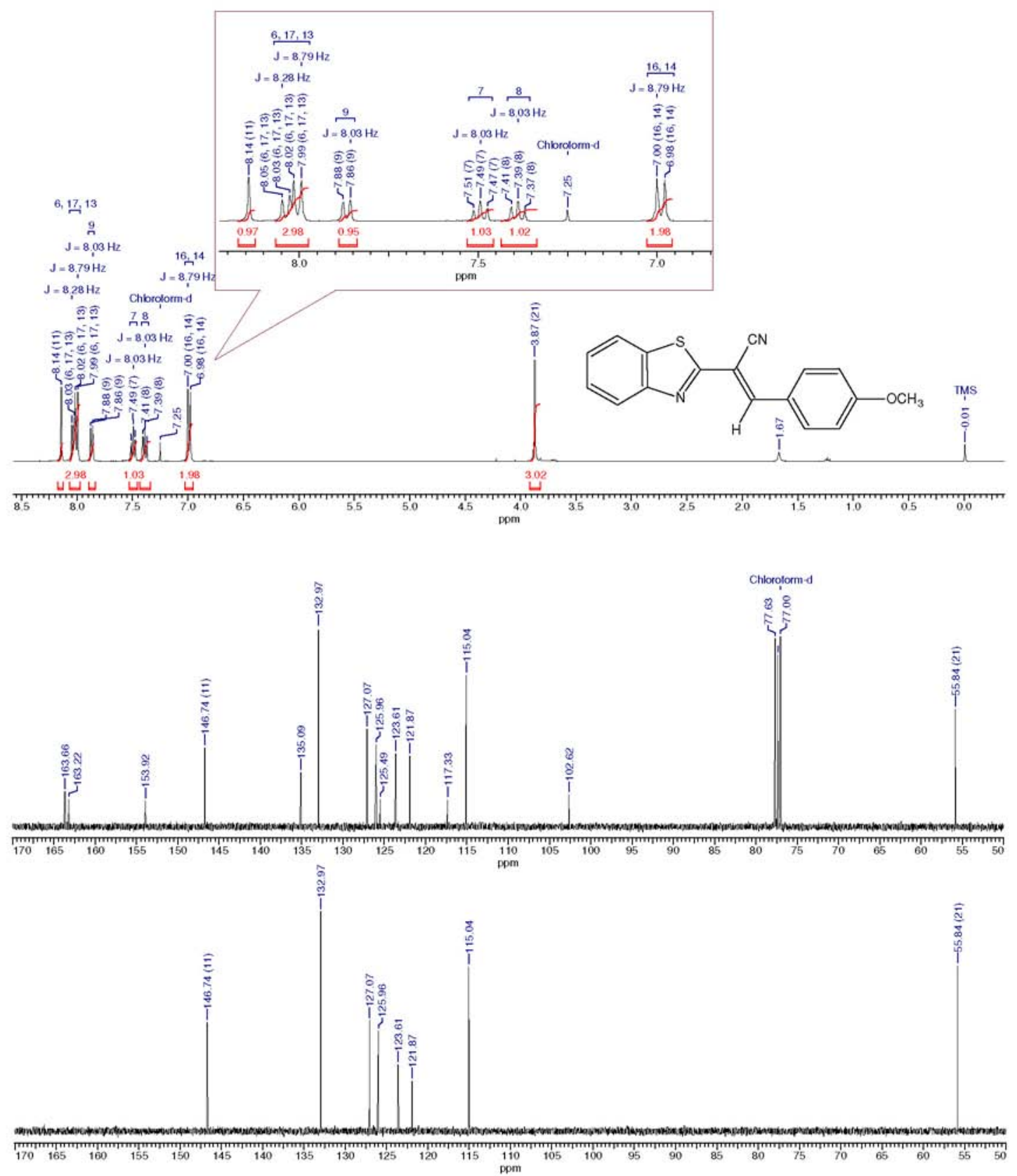

Figure S8. ${ }^{1} \mathrm{H},{ }^{13} \mathrm{C}$ and DEPT-135 spectra for compound $\mathbf{3 h}$. 

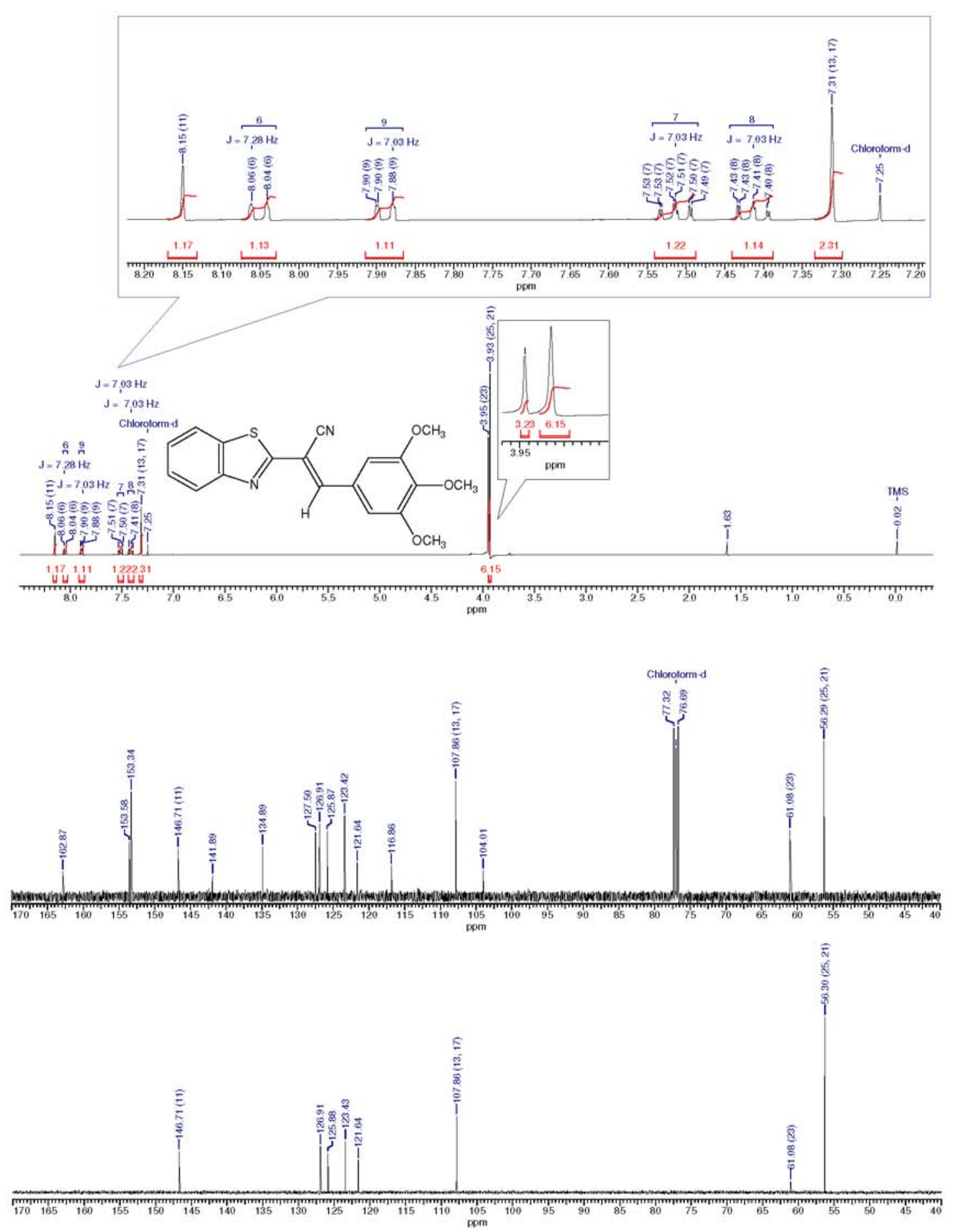

Figure S9. ${ }^{1} \mathrm{H},{ }^{13} \mathrm{C}$ and DEPT- 135 spectra for compound $\mathbf{3 i}$. 


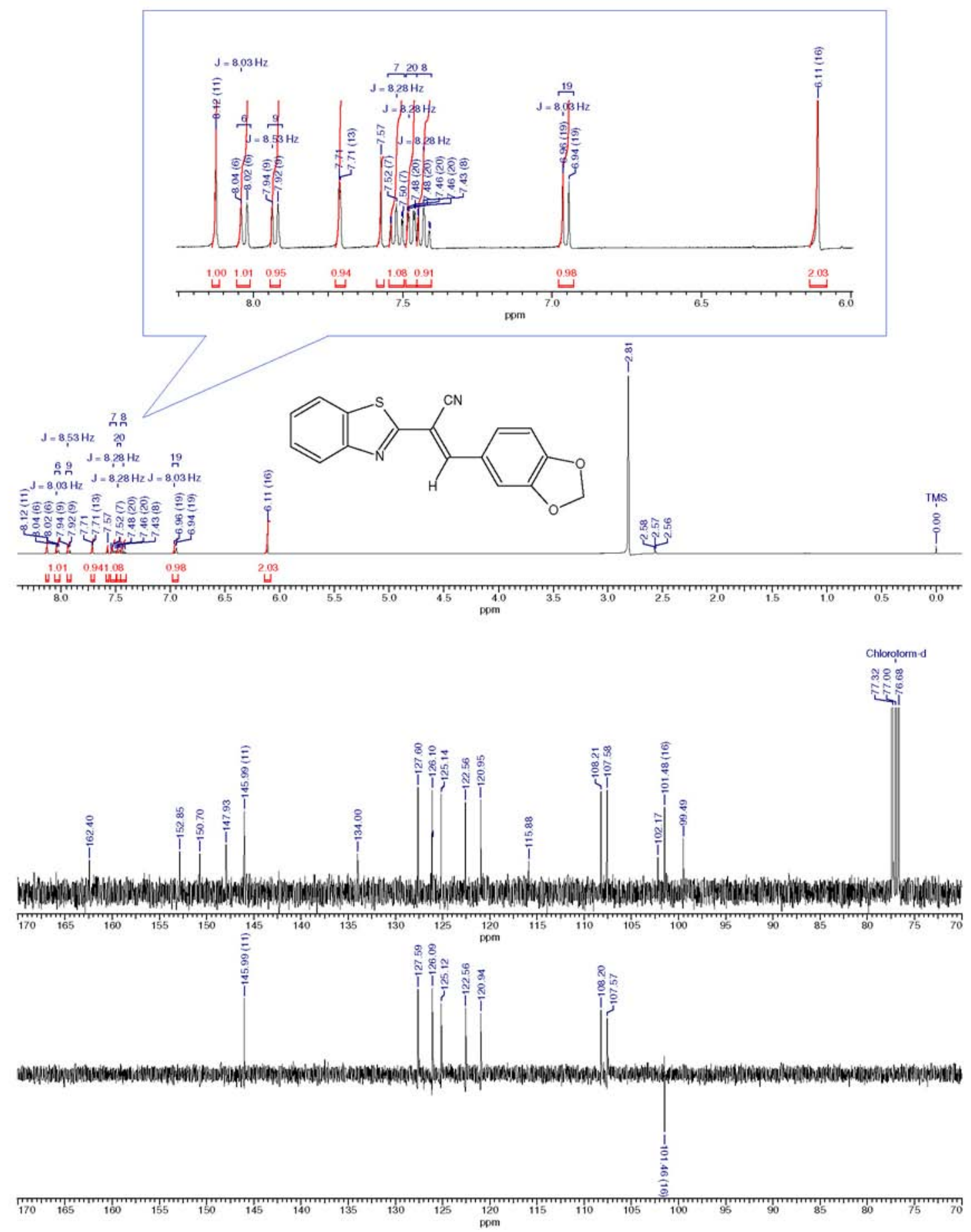

Figure S10. ${ }^{1} \mathrm{H},{ }^{13} \mathrm{C}$ and DEPT-135 spectra for compound $\mathbf{3 j}$. 

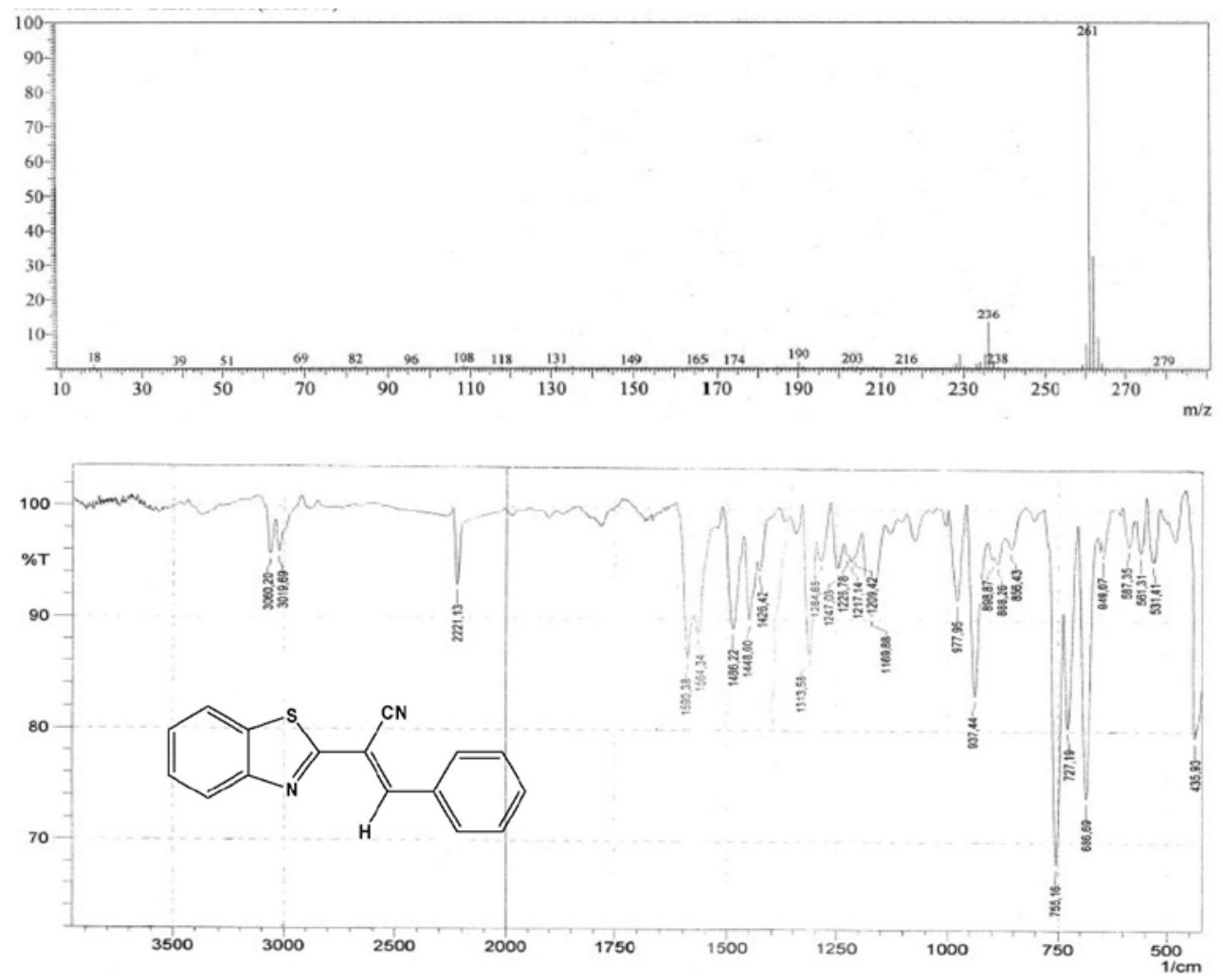

Figure S11. MS and IR spectra for Compound 3a.
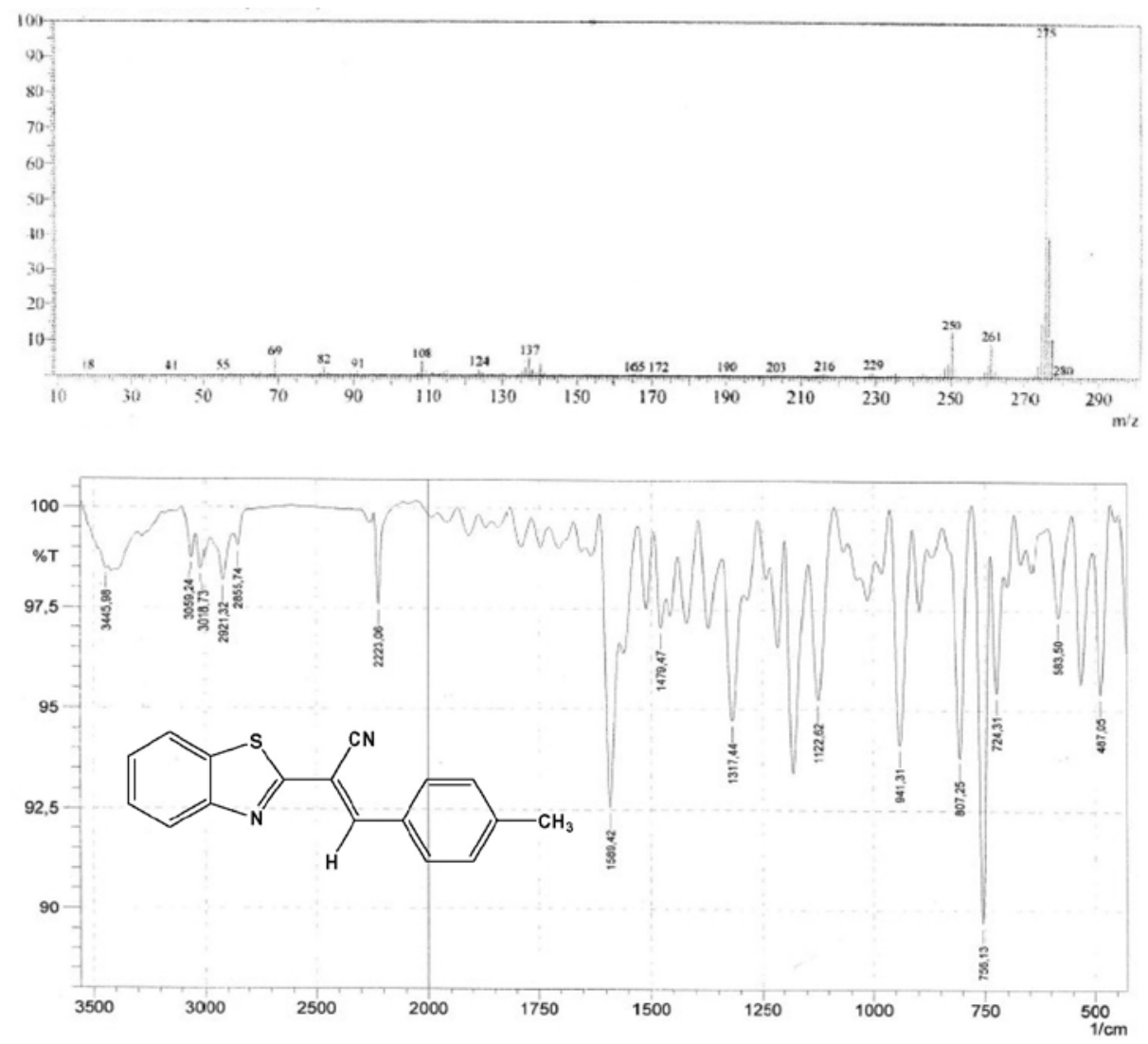

Figure S12. MS and IR spectra for Compound 3b. 

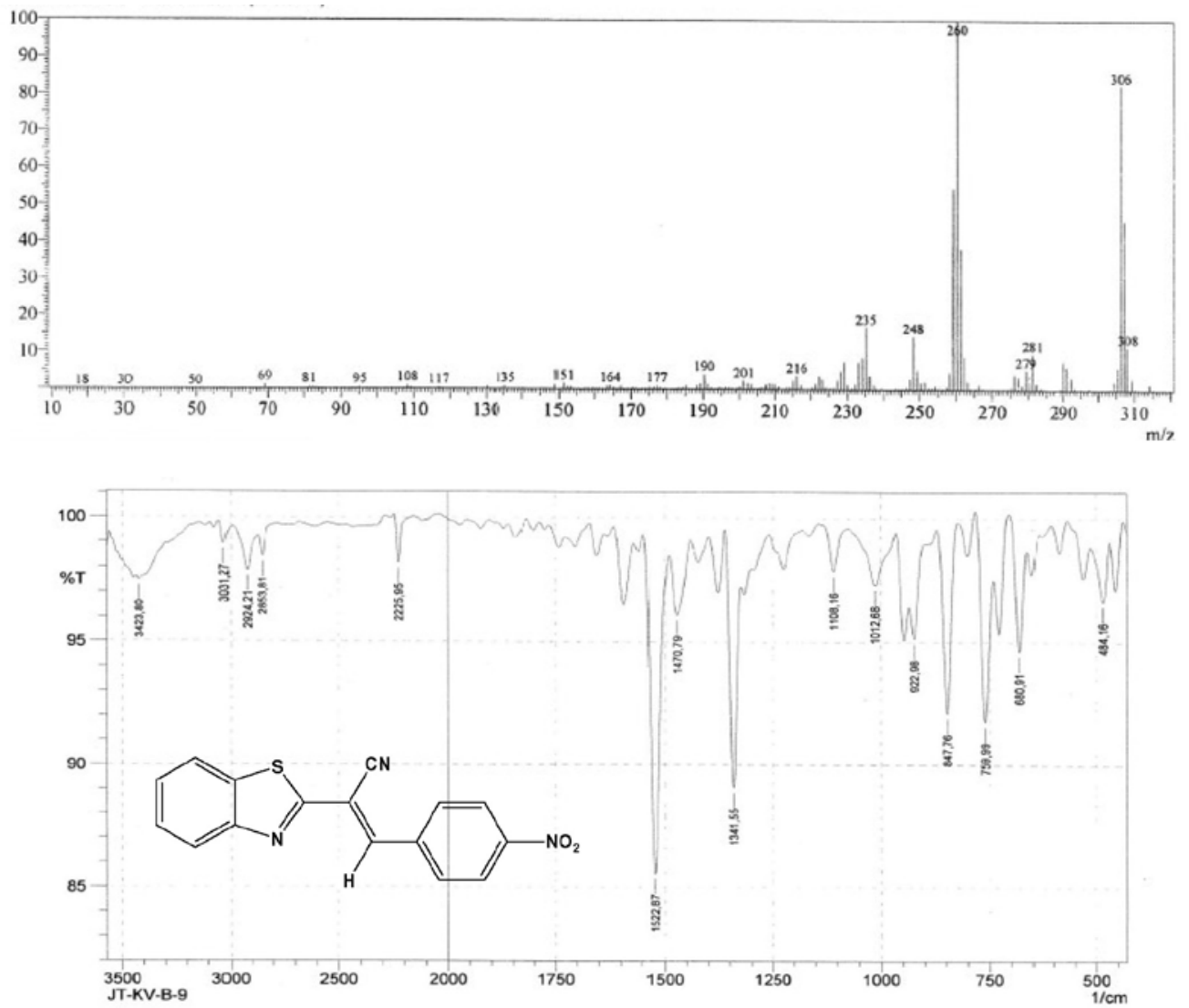

Figure S13. MS and IR spectra for compound 3c.
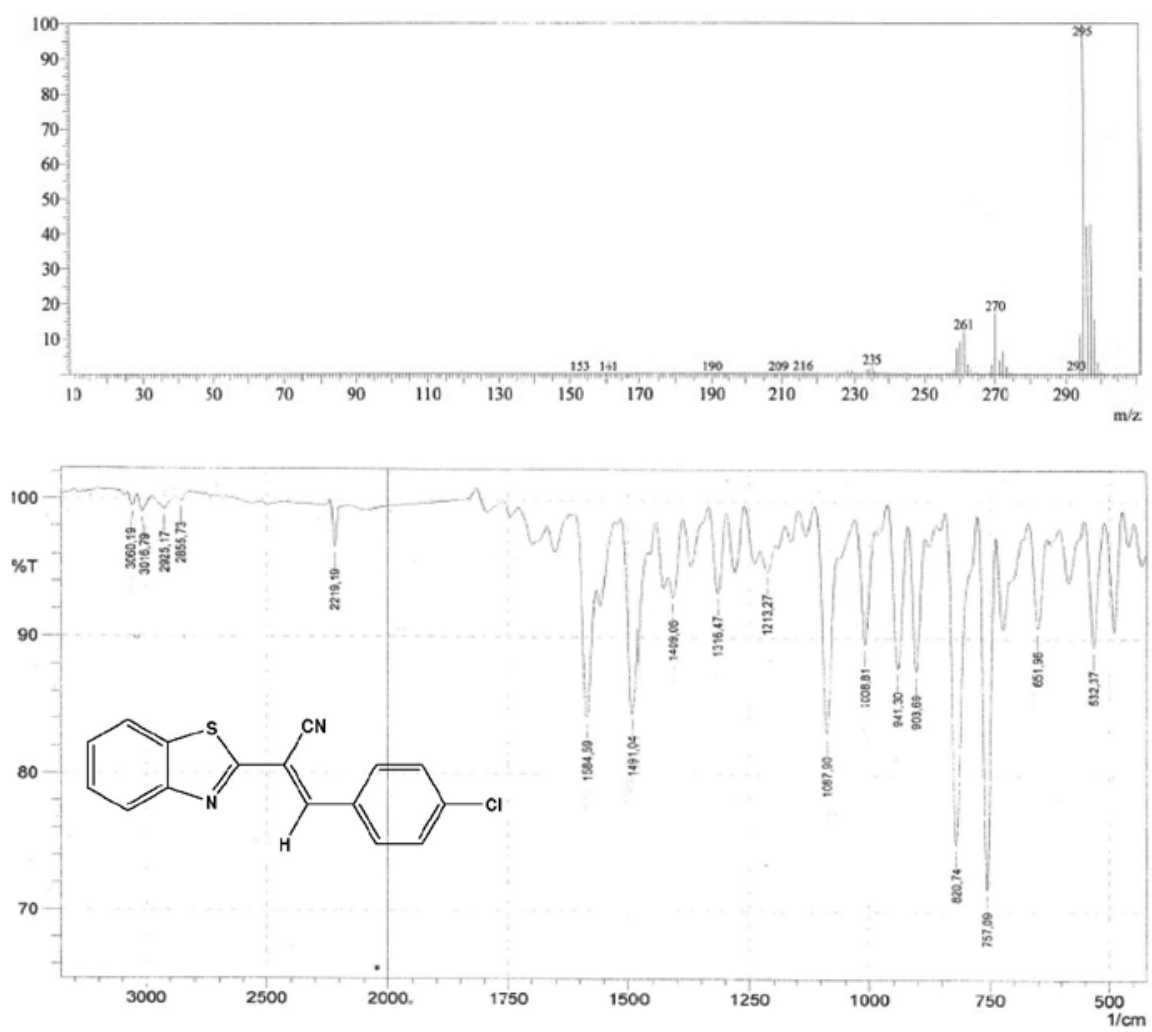

Figure S14. MS and IR spectra for compound 3d. 

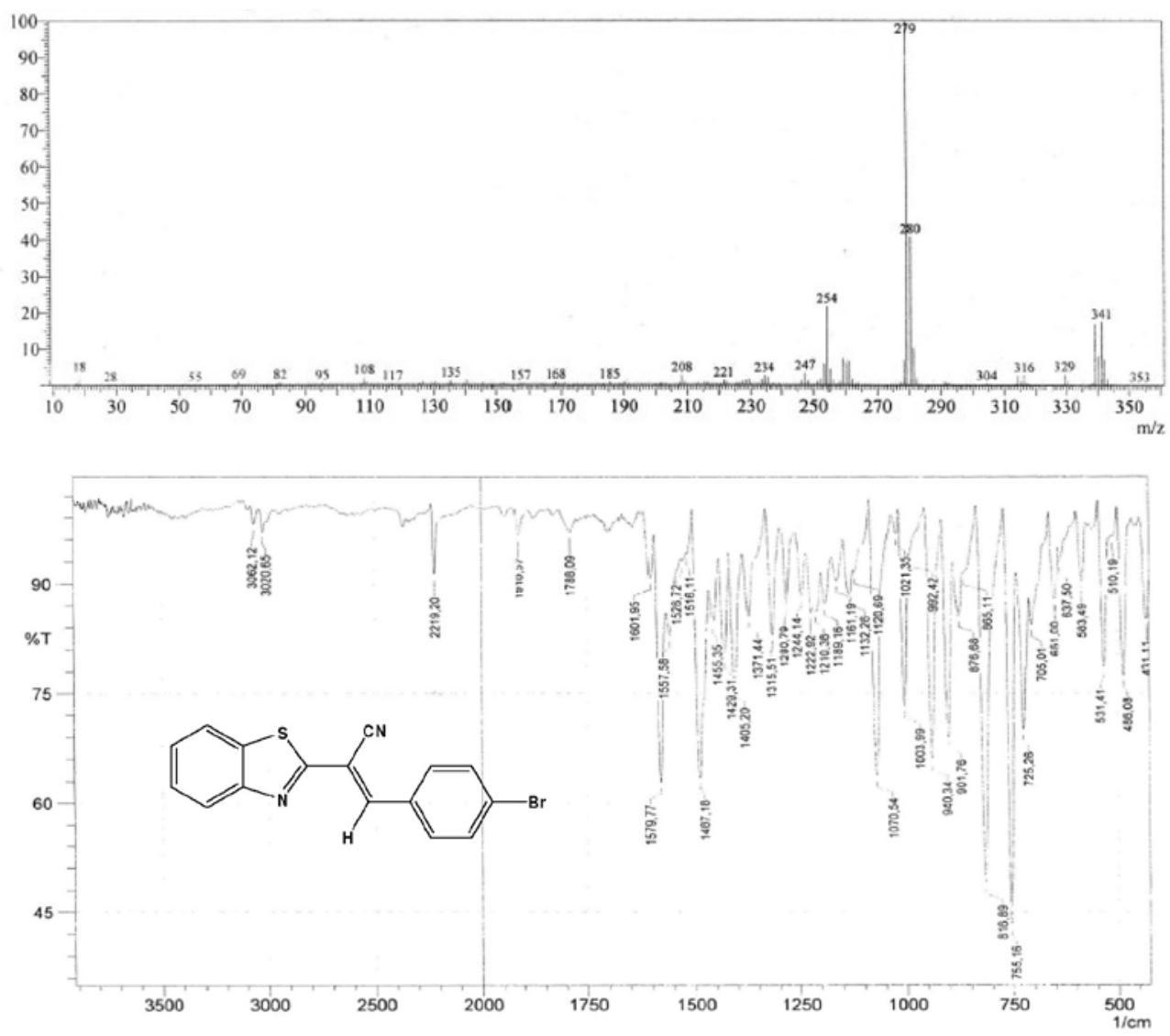

Figure S15. MS and IR spectra for compound 3e.
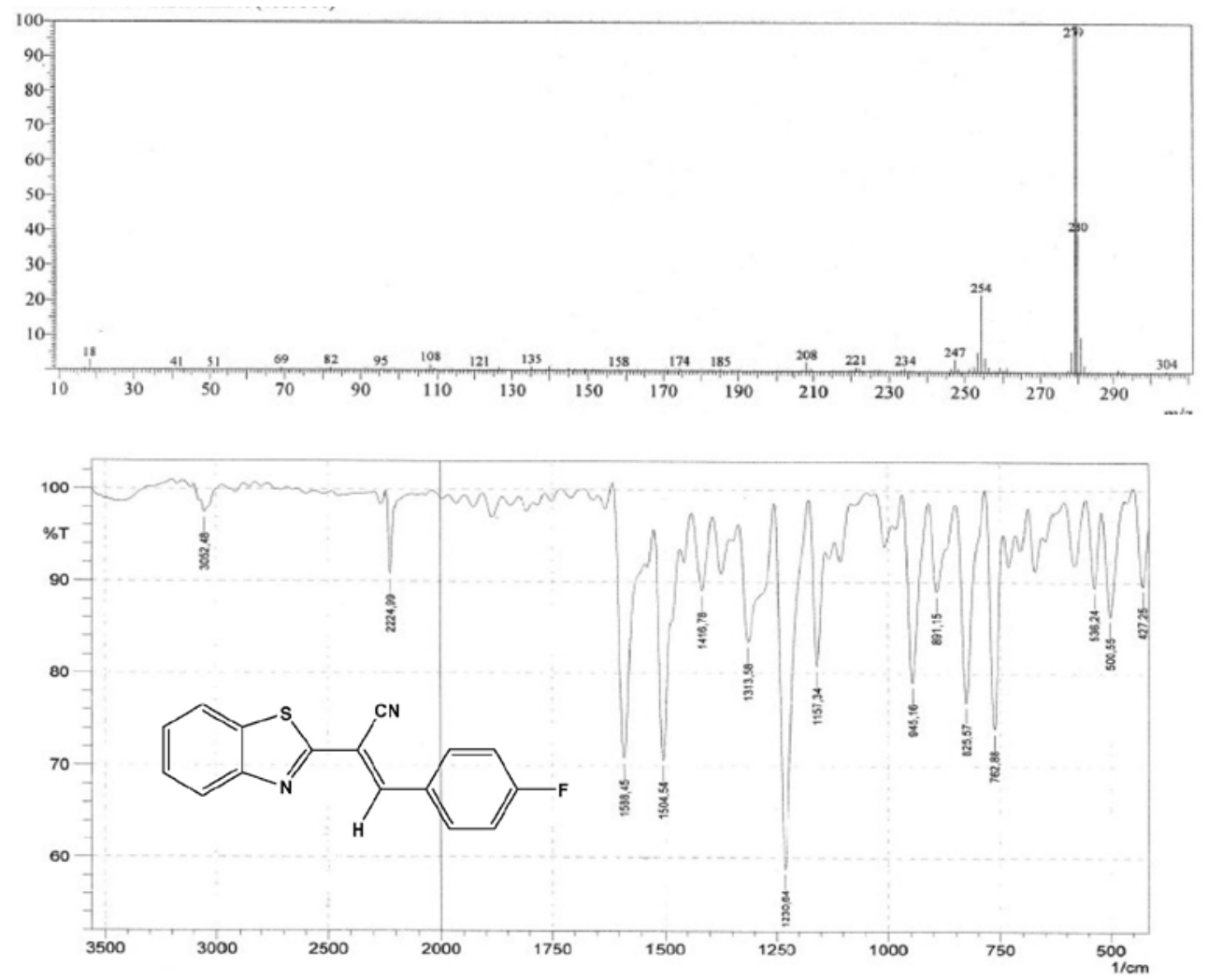

Figure S16. MS and IR spectra for compound $3 f$. 

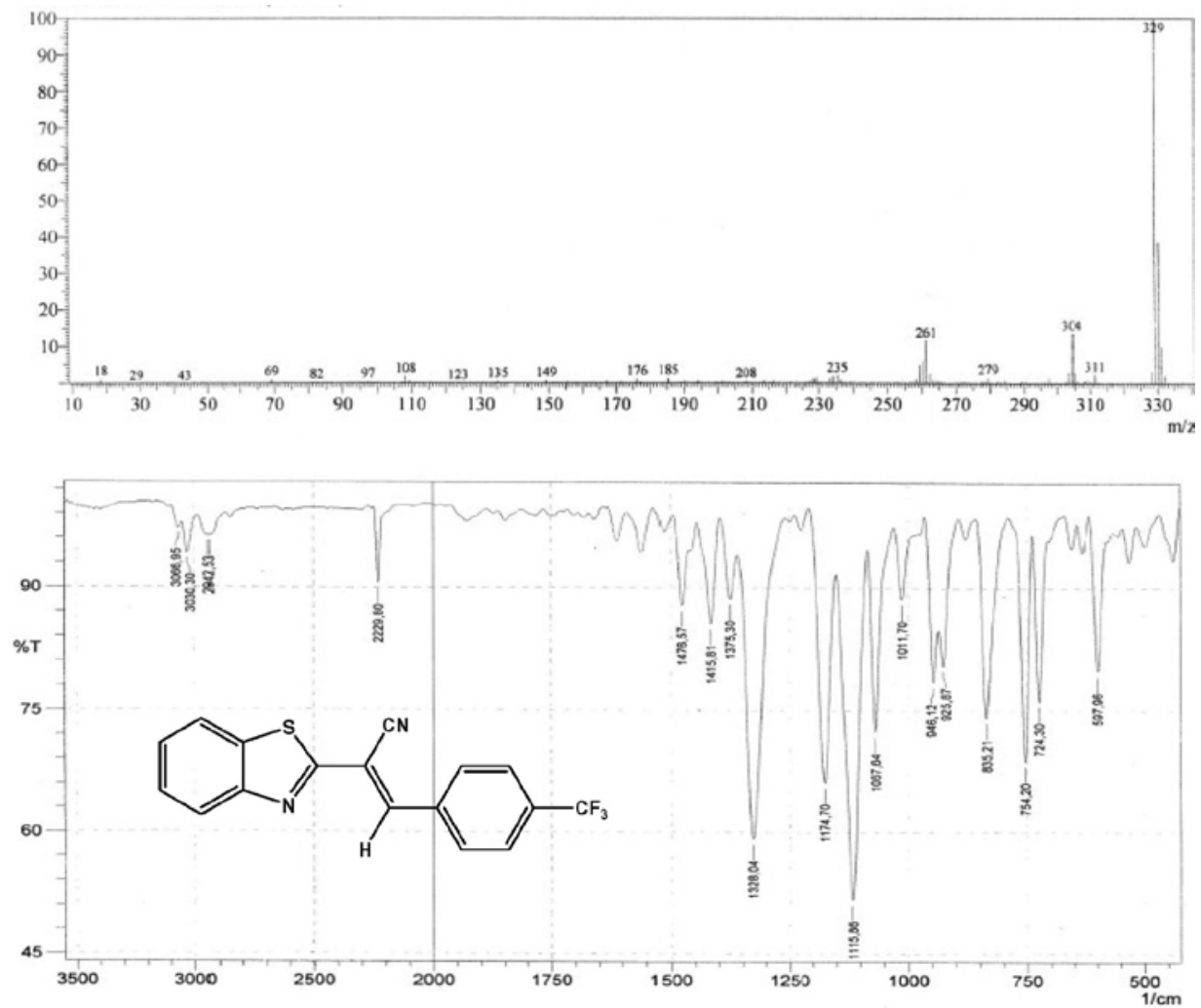

Figure S17. MS and IR spectra for compound 3g.
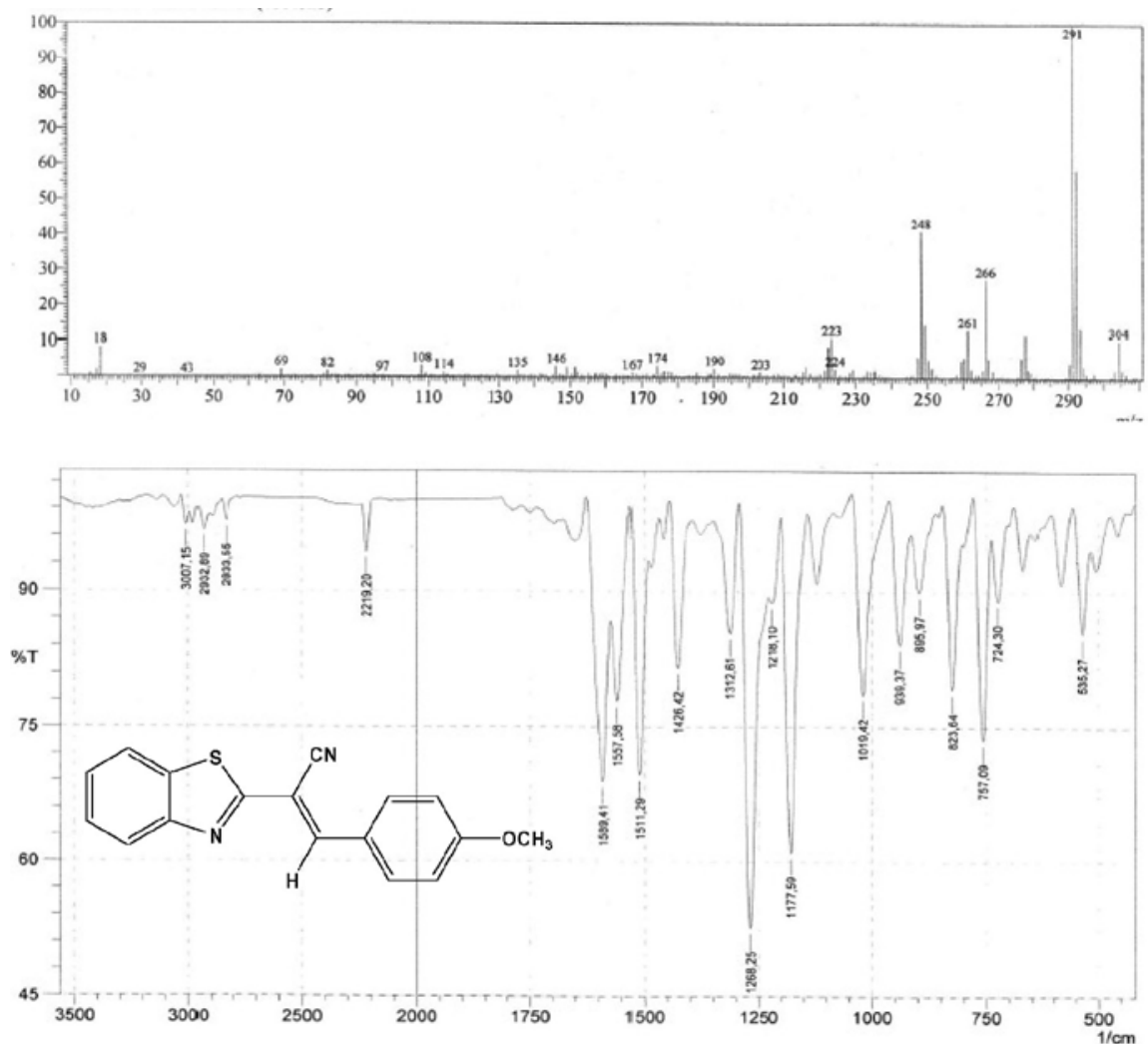

Figure S18. MS and IR spectra for compound $\mathbf{3 h}$. 

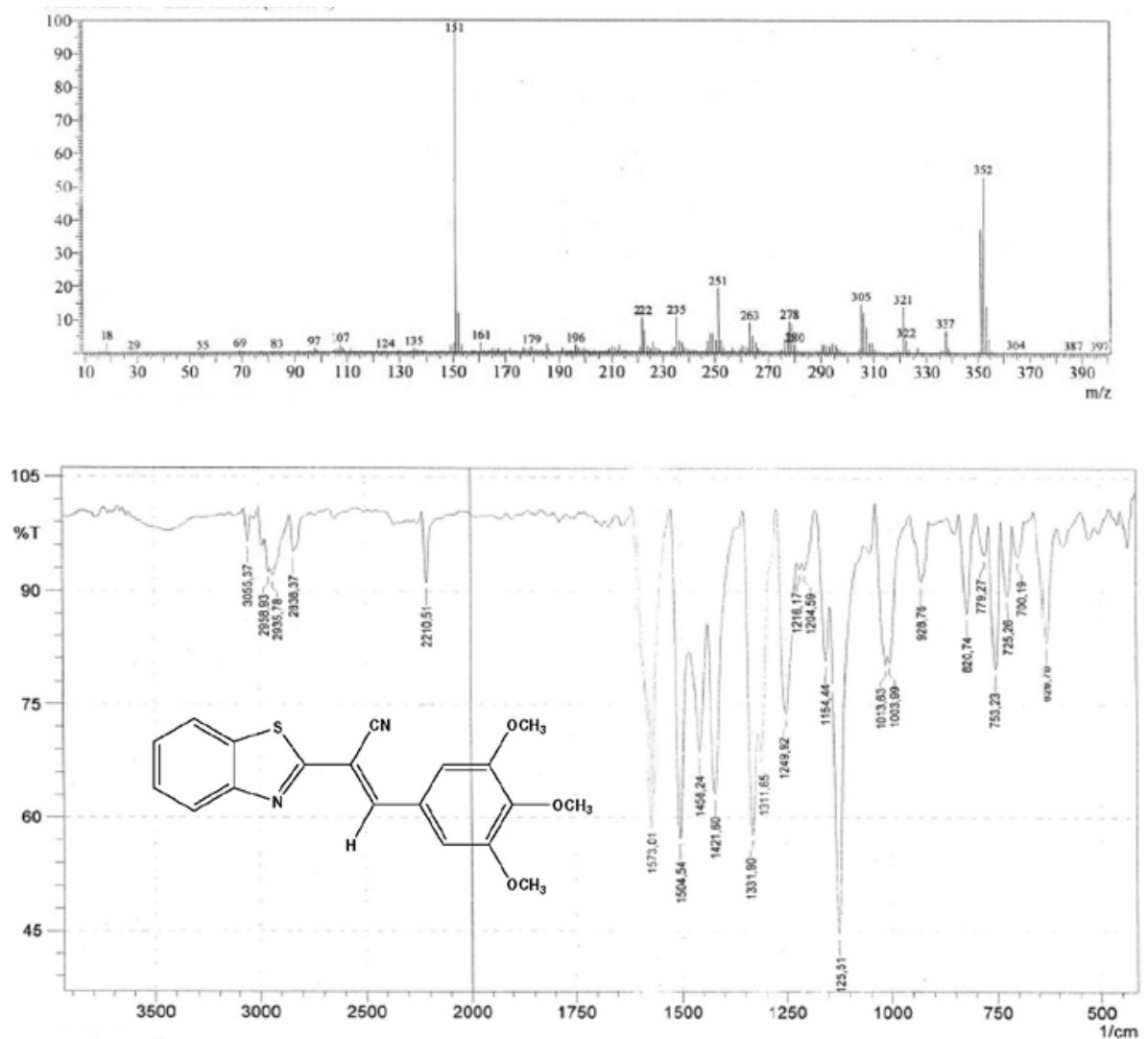

Figure S19. MS and IR spectra for compound 3i.
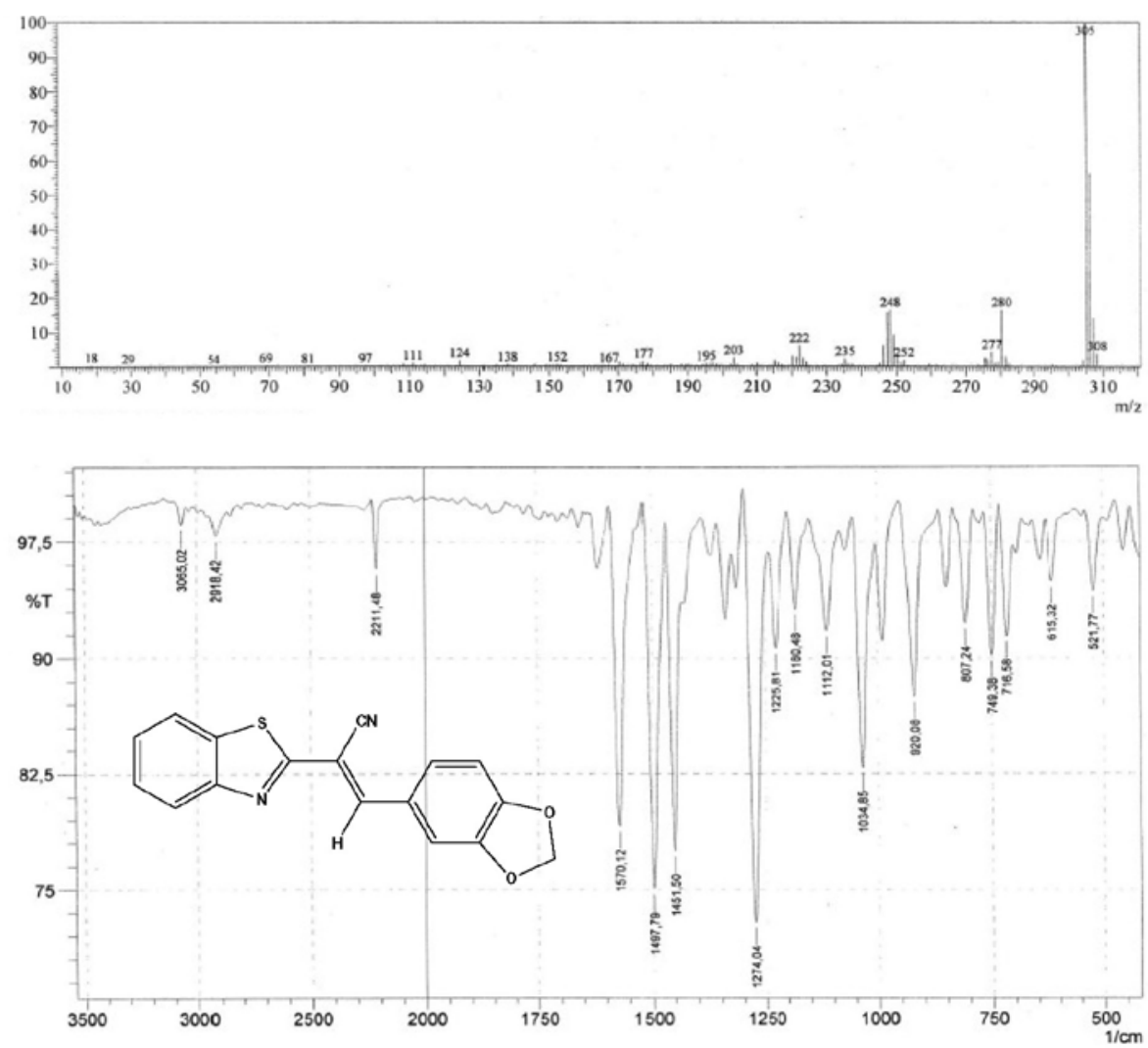

Figure S20. MS and IR spectra for compound 3j. 